\title{
Semialigned two Higgs doublet model
}

\author{
Naoyuki Haba, ${ }^{*}$ Hiroyuki Umeeda, ${ }^{\dagger}$ and Toshifumi Yamada \\ Graduate School of Science and Engineering, Shimane University, Matsue 690-8504, Japan
}

(Received 17 November 2017; published 5 February 2018)

\begin{abstract}
In the left-right symmetric model based on $S U(2)_{L} \times S U(2)_{R} \times U(1)_{B-L}$ gauge symmetry, there appear heavy neutral scalar particles mediating quark flavor changing neutral currents (FCNCs) at tree level. We consider a situation where such FCNCs give the only sign of the left-right model while $W_{R}$ gauge boson is decoupled, and name it "semialigned two Higgs doublet model" because the model resembles a two Higgs doublet model with mildly aligned Yukawa couplings to quarks. We predict a correlation among processes induced by quark FCNCs in the model, and argue that future precise calculation of meson-antimeson mixings and $C P$ violation therein may hint at the semialigned two Higgs doublet model and the left-right model behind it.
\end{abstract}

DOI: 10.1103/PhysRevD.97.035004

\section{INTRODUCTION}

The left-right symmetric model [1] based on $S U(2)_{L} \times$ $S U(2)_{R} \times U(1)_{B-L}$ gauge symmetry and the left-right parity (symmetry under the Lorentzian parity transformation accompanied by the exchange of $S U(2)_{L}$ and $S U(2)_{R}$ ) is a well-motivated extension of the standard model (SM). In the model, the chiral nature of the SM is beautifully attributed to spontaneous breaking of $S U(2)_{R} \times U(1)_{B-L}$ gauge symmetry. More importantly, the model offers a new look into the strong $C P$ problem; since the left-right parity demands the Yukawa couplings to be Hermitian and forbids gluon $\theta$ term at tree level, if one could find a symmetry-based reason that the two vacuum expectation values (VEVs) of the $S U(2)_{L} \times S U(2)_{R}$ bi-fundamental scalar are both made real, the strong $C P$ problem would be solved. Put another way, the original strong $C P$ problem, which is about a miraculous cancellation between the $\theta$ term in QCD and the quark mass phases in the theory of electroweak symmetry breaking, is simplified to the issue of why the scalar potential does not break $C P$ spontaneously. Thus, the strong $C P$ problem becomes more tractable, although the left-right model by itself does not solve it.

The first experimental hint of the left-right model would probably come in the form of quark flavor changing neutral currents (FCNCs) mediated by heavy neutral scalar particles

\footnotetext{
"haba@ riko.shimane-u.ac.jp †umeeda@riko.shimane-u.ac.jp

toshifumi@ riko.shimane-u.ac.jp
}

Published by the American Physical Society under the terms of the Creative Commons Attribution 4.0 International license. Further distribution of this work must maintain attribution to the author(s) and the published article's title, journal citation, and DOI. Funded by SCOAP . at tree level, because the $S U(2)_{L} \times S U(2)_{R}$ bi-fundamental scalar necessarily has two unaligned Yukawa couplings both contributing to up and down-type quark masses to accommodate the Cabibbo-Kobayashi-Maskawa (CKM) matrix, and the resultant quark FCNCs and $C P$ violation are efficiently searched for through meson-antimeson mixings [2-5]. On the other hand, recent studies have centered on the possibility of a direct measurement of $W_{R}$ gauge boson at the LHC, by elaborating a scalar potential where $S U(2)_{R} \times$ $U(1)_{B-L}$ breaking VEV $v_{R}$ is below $\sim 5 \mathrm{TeV}$ (hence $W_{R}$ mass being several $\mathrm{TeV}$ ), while the heavy scalar particles have masses above $\sim 20 \mathrm{TeV}$ to evade the bounds on FCNCs $[2,3]$. Such a scalar potential contains $\mathcal{O}(1)$ quartic couplings that quickly become nonperturbative along renormalization group (RG) evolutions [6,7], and even if this is circumvented, there is no theoretical reason that favors having specially heavy scalar particles. Conversely, if $W_{R}$ gauge boson has a mass similar to or larger than the heavy scalar particles [e.g., ( $W_{R}$ mass $) \gtrsim$ (heavy scalar mass) $\gtrsim$ $20 \mathrm{TeV}$, so that the model evades the constraints from meson-antimeson mixings], then $W_{R}$ gauge boson leaves no direct or indirect experimental signature and it is the heavy scalar particles that allow us to probe the left-right model through FCNCs they mediate. ${ }^{1}$

In this paper, therefore, we pose the following question: If quark FCNCs mediated by the heavy neutral scalars give the only sign of the left-right model, can we test the model? To answer this, we extract the bifundamental scalar part of the model, assuming that $W_{R}$ gauge boson is decoupled from phenomenology, and systematically study its indirect signatures and their correlation. We coin the term "semialigned two Higgs doublet model (semialigned 2HDM)" to

\footnotetext{
${ }^{1}$ For a collider study of the heavy scalar particles in the leftright model, see Ref. [8].
} 
describe the bifundamental scalar part, in light of the fact that the bi-fundamental scalar contains two $S U(2)_{L}$ doublet scalars and their quark Yukawa couplings are mildly aligned reflecting the smallness of CKM mixing angles.

Our analysis starts by realizing that the flavor and $\mathrm{CP}$ violating couplings of the heavy scalar particles are uniquely determined as follows: Since the search for neutron electric dipole moment (EDM) has put a severe bound on the strong $C P$ phase, we may concentrate on the limit with a vanishing spontaneous $C P$ phase for the bi-fundamental scalar VEVs [9]. ${ }^{2}$ In this limit, the quark mass matrices are Hermitian and the mixing matrix for right-handed quarks is identical with the CKM matrix, which allows one to express the couplings of the heavy scalar particles to quarks in terms of the SM quark masses and CKM matrix, without free parameters. The amplitudes for various FCNC processes are then calculated as functions of just one free parameter, that is, the nearly degenerate mass of the heavy scalar particles, and we thus predict a correlation among various FCNC processes. We will show that indirect $C P$ violation in kaon system, $\operatorname{Re} \epsilon$, the $B_{d}^{0}$ mass splitting, $\Delta M_{B_{d}}$, and the $B_{s}^{0}$ mass splitting, $\Delta M_{B_{s}}$, are the most sensitive probes for the semialigned 2HDM, and if uncertainties in their calculation are reduced and the prediction including the contributions of heavy scalar particles converges to the experimental values for some unique value of the heavy scalar mass, it is evidence for the semialigned $2 \mathrm{HDM}$ and the left-right model behind it.

A novelty of our study compared to previous works $[2,3]$ is that we concentrate on the limit with decoupled $W_{R}$ gauge boson, which enables us to compute all amplitudes with only one free parameter and investigate their correlation. Also, we pay attention to the fact that new physics contributions can distort the determination of the CKM matrix. To avoid this, when we derive the current bound on the semialigned 2HDM, we refit the CKM matrix, attempting to fit the experimental data with $\mathrm{SM}+$ new physics contributions and using a tension in the fitting to constrain the model. When we make a prediction for FCNC processes, we assume that the CKM matrix is determined beforehand in a way unaffected by new physics contributions.

This paper is organized as follows: In Sec. II, we describe the semialigned 2HDM induced from the leftright model, and calculate the mass and couplings of the heavy scalar particles. In Sec. III, we review the procedure for computing amplitudes for $\Delta F=2$ processes. In Sec. IV, we derive the current bound on the heavy neutral scalar mass. Section $\mathrm{V}$ presents our main results, which are $\operatorname{Re} \epsilon, \Delta M_{B_{d}}$ and $\Delta M_{B_{s}}$ expressed in terms of one parameter. Section VI is devoted to summary and discussion.

\footnotetext{
${ }^{2}$ In this paper, we focus on phenomenological consequences of a vanishing spontaneous $C P$ phase and do not discuss its theoretical origin. For attempts to derive the vanishing spontaneous $C P$ phase in the framework of the left-right model, see Ref. [10].
}

\section{MODEL}

We start from the minimal left-right symmetric model based on $S U(3)_{C} \times S U(2)_{L} \times S U(2)_{R} \times U(1)_{B-L}$ gauge symmetry [1] and the left-right parity, whose full expression is in the Appendix. The VEV of the $S U(2)_{R}$ triplet scalar, $v_{R}$, breaks $S U(2)_{R} \times U(1)_{B-L}$ into hypercharge $U(1)_{Y}$ and also breaks the left-right parity. Since the left-right parity dictates that the $S U(2)_{L}$ and $S U(2)_{R}$ gauge couplings be equal, and since one $S U(2)_{R}$ triplet scalar with $B-L=2$ is responsible for $S U(2)_{R} \times U(1)_{B-L} \rightarrow$ $U(1)_{Y}$ breaking, the masses of $W_{R}$ and $Z_{R}$ gauge bosons simply read $M_{W_{R}} \simeq g_{2} v_{R}$ and $M_{Z_{R}} \simeq \sqrt{2 g_{2}^{2}+2 g_{B L}^{2}} v_{R}$, respectively, with $g_{2}$ denoting the SM weak gauge coupling and $g_{B L}$ the $U(1)_{B-L}$ gauge coupling. In this paper, we focus on a limit where $v_{R}$ is above $20 \mathrm{TeV}$, and at the same time, the quartic couplings between two $S U(2)_{R}$ triplets and two bifundamentals are much smaller than 1 , namely,

$$
v_{R}>20 \mathrm{TeV}, \quad\left|\alpha_{1}\right|,\left|\alpha_{2 R}\right|,\left|\alpha_{2 I}\right|,\left|\alpha_{3}\right| \ll 1,
$$

where $\alpha_{1}, \alpha_{2 R}, \alpha_{2 I}, \alpha_{3}$ are defined in the Lagrangian of the Appendix. In the limit with Eq. (1), the low-energy theory at scales below $g_{2} v_{R}$ (we call it the semialigned 2HDM) contains the SM fermions + three right-handed neutrinos + the bifundamental scalar and possesses SM $S U(3)_{C} \times S U(2)_{L} \times U(1)_{Y}$ gauge symmetry. However, the Yukawa couplings and self-couplings of the bifundamental scalar respect global $S U(2)_{R}$ symmetry and the left-right parity, which are remnants of the left-right model, and hence have highly constrained structures. In Table I, we summarize the fields in the semialigned $2 \mathrm{HDM}$ and their charges in $S U(3)_{C} \times S U(2)_{L} \times U(1)_{Y}$ gauge group and global $S U(2)_{R}$ group. Here, the SM right-handed fermions form doublets of global $S U(2)_{R}$ symmetry, $q_{R}$ and $\ell_{R}$. The bifundamental scalar $\Phi$ is expressed as a $2 \times 2$ matrix transforming under a $S U(2)_{L} \times S U(2)_{R}$ gauge transformation as

$$
\begin{aligned}
& \Phi \rightarrow e^{i \tau^{a} \theta_{L}^{a}} \Phi e^{-i \tau^{a} \theta_{R}^{a}}, \quad \theta_{L}^{a}, \theta_{R}^{a}: \text { gauge parameters, } \\
& \tau^{a} \equiv \sigma^{a} / 2,
\end{aligned}
$$

which is then decomposed into two $S U(2)_{L}$ doublet scalars with hypercharge $Y= \pm 1 / 2, H_{u}$ and $H_{d}$, as $\Phi=\left(i \sigma_{2} H_{u}^{*}, i \sigma_{2} H_{d}^{*}\right)$. The Lagrangian of the semialigned $2 \mathrm{HDM}$ is given by

$$
\begin{aligned}
-\mathcal{L}= & \left(Y_{q}\right)_{i j} \bar{q}_{L}^{i} \Phi q_{R}^{j}+\left(\tilde{Y}_{q}\right)_{i j} \bar{q}_{L}^{i} \tilde{\Phi} q_{R}^{j}+\left(Y_{\ell}\right)_{i j} \bar{\ell}_{L}^{i} \Phi \ell_{R}^{j} \\
& +\left(\tilde{Y}_{\ell}\right)_{i j} \bar{\ell}_{L}^{i} \tilde{\Phi} \ell_{R}^{j}+\text { H.c. } \\
& +\frac{v_{R}}{\sqrt{2}}\left(Y_{M}\right)_{i j} N_{R}^{i T} \epsilon N_{R}^{j}+\text { H.c. }
\end{aligned}
$$


TABLE I. Field content and charge assignments. $i=1,2,3$ is the flavor index.

\begin{tabular}{lccccc}
\hline \hline Field & Lorentz $S O(1,3)$ & $S U(3)_{C}$ & $S U(2)_{L}$ & global $S U(2)_{R}$ & $U(1)_{Y}$ \\
\hline$q_{L}^{i}$ & $(\mathbf{2}, \mathbf{1})$ & $\mathbf{3}$ & $\mathbf{2}$ & $\mathbf{1}$ & $1 / 6$ \\
$q_{R}^{i}=\left(\begin{array}{c}u_{R}^{i} \\
d_{R}^{i}\end{array}\right)$ & $(\mathbf{1}, \mathbf{2})$ & $\mathbf{3}$ & $\mathbf{1}$ & $\mathbf{2}$ & $\left(\begin{array}{c}2 / 3 \\
-1 / 3\end{array}\right)$ \\
$\ell_{L}^{i}$ & $(\mathbf{2}, \mathbf{1})$ & $\mathbf{1}$ & $\mathbf{2}$ & $\mathbf{1}$ & $-1 / 2$ \\
$\ell_{R}^{i}=\left(\begin{array}{c}v_{R}^{i} \\
e_{R}^{i}\end{array}\right)$ & $(\mathbf{1}, \mathbf{2})$ & $\mathbf{1}$ & $\mathbf{1}$ & $\mathbf{2}$ & $\left(\begin{array}{c}0 \\
-1\end{array}\right)$ \\
$\Phi=\left(i \sigma_{2} H_{u}^{*}, i \sigma_{2} H_{d}^{*}\right)$ & $\mathbf{1}$ & 1 & $\mathbf{2}$ & $\mathbf{2}$ & $(-1 / 2,1 / 2)$ \\
\hline \hline
\end{tabular}

$$
\begin{aligned}
& +m_{1}^{2} \operatorname{tr}\left[\Phi^{\dagger} \Phi\right]+m_{2 R}^{2} \operatorname{tr}\left[\Phi^{\dagger} \tilde{\Phi}+\Phi \tilde{\Phi}^{\dagger}\right]+m_{2 I}^{2} i \operatorname{tr}\left[\Phi^{\dagger} \tilde{\Phi}-\Phi \tilde{\Phi}^{\dagger}\right] \\
& +m_{3}^{2} \operatorname{tr}\left[\Phi^{\dagger} \Phi\left(\begin{array}{ll}
1 & 0 \\
0 & 0
\end{array}\right)\right] \\
& +\lambda_{1} \operatorname{tr}\left[\Phi^{\dagger} \Phi\right]^{2}+\lambda_{2}\left(\operatorname{tr}\left[\Phi^{\dagger} \tilde{\Phi}\right]^{2}+\operatorname{tr}\left[\Phi \tilde{\Phi}^{\dagger}\right]^{2}\right) \\
& +\lambda_{3} \operatorname{tr}\left[\Phi^{\dagger} \tilde{\Phi}\right] \operatorname{tr}\left[\Phi \tilde{\Phi}^{\dagger}\right]+\lambda_{4} \operatorname{tr}\left[\Phi^{\dagger} \Phi\right] \operatorname{tr}\left[\Phi^{\dagger} \tilde{\Phi}+\Phi \tilde{\Phi}^{\dagger}\right] \\
& \quad \text { with } \tilde{\Phi} \equiv i \sigma_{2} \Phi^{*} i \sigma_{2}
\end{aligned}
$$

where $m_{1}^{2}, m_{2 R}^{2}, m_{2 I}^{2}, m_{3}^{2}, \lambda_{1}, \lambda_{2}, \lambda_{3}, \lambda_{4}$ are all real, and the Yukawa coupling matrices are Hermitian, $Y_{q}^{\dagger}=Y_{q}$, $\tilde{Y}_{q}^{\dagger}=\tilde{Y}_{q}, Y_{\ell}^{\dagger}=Y_{\ell}, \tilde{Y}_{\ell}^{\dagger}=\tilde{Y}_{\ell}$. Notice that $m_{2 I}^{2}$ softly breaks the left-right parity, and $m_{3}^{2}$ softly breaks $S U(2)_{R}$ symmetry. Both result from the spontaneous left-right symmetry breaking and are proportional to $v_{R}^{2}$.

$\Phi$ develops a VEV to break $S U(2)_{L} \times U(1)_{Y}$ symmetry. Through a $S U(2)_{L}$ plus $\sigma_{3}$ part of $S U(2)_{R}$ symmetry transformation, ${ }^{3}$ the VEV is made into the following form, with one VEV having a $C P$ phase $\alpha$ :

$$
\begin{aligned}
\langle\Phi\rangle & =\frac{1}{\sqrt{2}}\left(\begin{array}{cc}
v \sin \beta & 0 \\
0 & -e^{i \alpha} v \cos \beta
\end{array}\right), \\
v & \simeq 246 \mathrm{GeV}, \quad \sin \beta>0, \quad \cos \beta>0 .
\end{aligned}
$$

The mass matrices for up-type quarks, $M_{u}$, down-type quarks, $M_{d}$, charged leptons, $M_{e}$, are given by

$$
\begin{aligned}
& M_{u}=\frac{v}{\sqrt{2}}\left(Y_{q} \sin \beta+\tilde{Y}_{q} \cos \beta e^{-i \alpha}\right), \\
& M_{d}=-\frac{v}{\sqrt{2}}\left(Y_{q} \cos \beta e^{i \alpha}+\tilde{Y}_{q} \sin \beta\right), \\
& M_{e}=-\frac{v}{\sqrt{2}}\left(Y_{\ell} \cos \beta e^{i \alpha}+\tilde{Y}_{\ell} \sin \beta\right) .
\end{aligned}
$$

The spontaneous $C P$ phase $\alpha$ has already been severely constrained by the search for neutron EDM. Since $W_{R}$ is decoupled and the coupling of scalar particles to up and down quarks is Yukawa-suppressed, perturbative

\footnotetext{
${ }^{3}$ This symmetry transformation generates a phase for $v_{R}$, but this can be negated by a $U(1)_{B-L}$ symmetry transformation.
}

corrections to neutron EDM are negligible, and the experimental bound is directly translated into a bound on $\arg \operatorname{det}\left(M_{u} M_{d}\right)$ and hence on $\alpha$. In the limit of neglecting the quark flavor mixing (but no assumptions are made on $\beta$ or $\alpha$ ), we obtain, from Eqs. (7), (8), the following formula:

$$
\begin{aligned}
\arg \operatorname{det}\left(M_{u} M_{d}\right) & \simeq \theta_{u d}+\theta_{c s}+\theta_{t b}, \\
\sin \theta_{u d} & =-\frac{m_{u}^{2}-m_{d}^{2}}{2 m_{u} m_{d}} \tan (2 \beta) \sin \alpha, \quad(u, d) \rightarrow(c, s), \\
(u, d) & \rightarrow(t, b) .
\end{aligned}
$$

The current experimental bound [11] roughly gives

$$
10^{-10}>|\bar{\theta}|=\left|\arg \operatorname{det}\left(M_{u} M_{d}\right)\right| \simeq\left|\theta_{u d}+\theta_{c s}+\theta_{t b}\right|,
$$

where it should be reminded that the QCD $\theta$ term is prohibited at tree level by the left-right parity. $\alpha$ is thus constrained to be much below 1 , and based on this fact, we fix $\alpha=0$ in the rest of the paper. ${ }^{4}$

The physical scalar particles after the electroweak symmetry breaking are ${ }^{5}$ a charged scalar, $H^{ \pm}$, a $C P$-odd scalar, $A$, a lighter $C P$-even scalar which we identify with the SM Higgs particle, $h$, and a heavier $C P$-even scalar, $H$. The $H^{ \pm}$and $A$ masses read

$$
\begin{gathered}
m_{H^{ \pm}}^{2}=\frac{m_{3}^{2}}{\cos 2 \beta}, \\
m_{A}^{2}=\frac{m_{3}^{2}}{\cos 2 \beta}-\left(4 \lambda_{2}-2 \lambda_{3}\right) v^{2},
\end{gathered}
$$

The $H$ and $h$ masses expanded to the order of $\mathcal{O}\left(v^{2} /\left|m_{3}^{2}\right|\right)$ are found to be

$$
m_{H}^{2} \simeq \frac{m_{3}^{2}}{\cos 2 \beta}+\left(4 \lambda_{2}+2 \lambda_{3}\right) \cos ^{2} 2 \beta v^{2},
$$

$m_{h}^{2} \simeq 2 \lambda_{1} v^{2}+\left(4 \lambda_{2}+2 \lambda_{3}\right) \sin ^{2} 2 \beta v^{2}+4 \lambda_{4} \sin 2 \beta v^{2}$.

\footnotetext{
${ }^{4}$ Deriving $\alpha=0$ theoretically is equivalent to solving the strong $C P$ problem, which we do not attempt in this paper.

${ }^{5}$ Since $\alpha=0, C P$ is not broken spontaneously and the $C P$ even and odd scalar particles can be defined.
} 
The bifundamental scalar $\Phi=\left(i \sigma_{2} H_{u}^{*}, i \sigma_{2} H_{d}^{*}\right)$ can be decomposed into the physical scalar particles $H^{ \pm}, A, H, h$ and Nambu-Goldstone bosons, $G^{ \pm}, G^{0}$, in the following way:

$$
\begin{aligned}
& H_{u}=\left(\begin{array}{c}
-\sin \beta G^{+}+\cos \beta H^{+} \\
\frac{1}{\sqrt{2}}\left(\sin \beta v+\cos \gamma h+\sin \gamma H-i \sin \beta G^{0}+i \cos \beta A\right)
\end{array}\right), \\
& H_{d}=\left(\begin{array}{c}
\frac{1}{\sqrt{2}}\left(\cos \beta v-\sin \gamma h+\cos \gamma H+i \cos \beta G^{0}+i \sin \beta A\right) \\
\cos \beta G^{-}+\sin \beta H^{-}
\end{array}\right),
\end{aligned}
$$

where $\gamma$ is the mixing angle of the $C P$-even scalars satisfying

$$
\tan 2 \gamma=\frac{m_{3}^{2}-2 v^{2}\left(\lambda_{1}+2 \lambda_{2}+\lambda_{3}\right) \cos 2 \beta-4 v^{2} \lambda_{4} \cot 2 \beta}{m_{3}^{2}-2 v^{2}\left(\lambda_{1}-2 \lambda_{2}-\lambda_{3}\right) \cos 2 \beta} \tan 2 \beta, \quad 0>\gamma>-\pi / 2 .
$$

Since the $A$ and $H$ masses are experimentally constrained to be above $\sim 10 \mathrm{TeV}$, we work in the decoupling limit with $v^{2} /\left|m_{3}^{2}\right| \rightarrow 0$ to realize $m_{H}^{2}, m_{A}^{2} \gg m_{h}^{2}$, in which case the masses of $H^{ \pm}, A, H$ and the $C P$-even scalar mixing angle $\gamma$ satisfy

$$
\begin{gathered}
m_{H^{ \pm}}^{2}=m_{A}^{2}=m_{H}^{2}, \\
\gamma=\beta-\frac{\pi}{2} .
\end{gathered}
$$

Equation (A4) induces Yukawa couplings for quarks and $H^{ \pm}, H$ and $A$, given by

$$
\begin{aligned}
-\mathcal{L}_{\text {yukawa }}= & \left(Y_{q} \sin \beta-\tilde{Y}_{q} \cos \beta\right)_{i j} \bar{u}_{L}^{i} d_{R}^{j} H^{+}+\left(-Y_{q} \cos \beta+\tilde{Y}_{q} \sin \beta\right)_{i j} \bar{d}_{L}^{i} u_{R}^{j} H^{-}+\text {H.c. } \\
& +\frac{i}{\sqrt{2}}\left(-Y_{q} \cos \beta+\tilde{Y}_{q} \sin \beta\right)_{i j} \bar{u}^{i} \gamma_{5} u^{j} A+\frac{i}{\sqrt{2}}\left(Y_{q} \sin \beta-\tilde{Y}_{q} \cos \beta\right)_{i j} \bar{d}^{i} \gamma_{5} d^{j} A \\
& +\frac{1}{\sqrt{2}}\left(Y_{q} \sin \gamma+\tilde{Y}_{q} \cos \gamma\right)_{i j} \bar{u}^{i} u^{j} H+\frac{1}{\sqrt{2}}\left(-Y_{q} \cos \gamma-\tilde{Y}_{q} \sin \gamma\right)_{i j} \bar{d}^{i} d^{j} H \\
\simeq & -\sqrt{2} \frac{\left(M_{u}+M_{d} \sin 2 \beta\right)_{i j}}{v \cos 2 \beta} \bar{u}_{L}^{i} d_{R}^{j} H^{+}+\sqrt{2} \frac{\left(M_{d}+M_{u} \sin 2 \beta\right)_{i j}}{v \cos 2 \beta} \bar{d}_{L}^{i} u_{R}^{j} H^{-}+\text {H.c. } \\
& +\frac{\left(M_{d}+M_{u} \sin 2 \beta\right)_{i j}}{v \cos 2 \beta} \bar{u}^{i} u^{j} H+i \frac{\left(M_{d}+M_{u} \sin 2 \beta\right)_{i j}}{v \cos 2 \beta} \bar{u}^{i} \gamma_{5} u^{j} A \\
& +\frac{\left(M_{u}+M_{d} \sin 2 \beta\right)_{i j}}{v \cos 2 \beta} \bar{d}^{i} d^{j} H-i \frac{\left(M_{u}+M_{d} \sin 2 \beta\right)_{i j}}{v \cos 2 \beta} \bar{d}^{i} \gamma_{5} d^{j} A,
\end{aligned}
$$

where Eq. (19) has been used. We diagonalize the quark mass matrices by the rotation,

$$
u_{L, R} \rightarrow V_{u} u_{L, R}, \quad d_{L, R} \rightarrow V_{d} d_{L, R},
$$

to obtain

$$
V_{u}^{\dagger} M_{u} V_{u}=m_{u}^{D}, \quad V_{d}^{\dagger} M_{d} V_{d}=m_{d}^{D},
$$

$$
\begin{aligned}
& m_{u}^{D}=\operatorname{diag}\left(s_{u} m_{u}, s_{c} m_{c}, s_{t} m_{t}\right), \\
& m_{d}^{D}=\operatorname{diag}\left(s_{d} m_{d}, s_{s} m_{s}, s_{b} m_{b}\right),
\end{aligned}
$$

where $m_{u}^{D}$ and $m_{d}^{D}$ are diagonalized mass matrices for up and down-type quarks, respectively, and $s_{f}= \pm 1(f=u, c, t, d, s, b)$, which reflects sign uncertainty of the mass eigenvalues. Note that the left and right-handed quarks are rotated with the same unitary matrix, since $\alpha=0$ and the mass matrices are Hermitian. Accordingly, the Yukawa couplings become 


$$
\begin{aligned}
-\mathcal{L}_{\text {yukawa }}= & -\sqrt{2} \frac{\left(m_{u}^{D} V+V^{\dagger} m_{d}^{D} \sin 2 \beta\right)_{i j}}{v \cos 2 \beta} \bar{u}_{L}^{i} d_{R}^{j} H^{+} \\
& +\sqrt{2} \frac{\left(m_{d}^{D} V^{\dagger}+V m_{u}^{D} \sin 2 \beta\right)_{i j}}{v \cos 2 \beta} \bar{d}_{L}^{i} u_{R}^{j} H^{-}+\text {H.c. } \\
& +\frac{\left(V m_{d}^{D} V^{\dagger}+m_{u}^{D} \sin 2 \beta\right)_{i j}}{v \cos 2 \beta} \bar{u}^{i} u^{j} H \\
& +i \frac{\left(V m_{d}^{D} V^{\dagger}+m_{u}^{D} \sin 2 \beta\right)_{i j}}{v \cos 2 \beta} \bar{u}^{i} \gamma_{5} u^{j} A \\
& +\frac{\left(V^{\dagger} m_{u}^{D} V+m_{d}^{D} \sin 2 \beta\right)_{i j}}{v \cos 2 \beta} \bar{d}^{i} d^{j} H \\
& -i \frac{\left(V^{\dagger} m_{u}^{D} V+m_{d}^{D} \sin 2 \beta\right)_{i j}}{v \cos 2 \beta} \bar{d}^{i} \gamma_{5} d^{j} A
\end{aligned}
$$

where we have defined the CKM matrix, $V$, as

$$
V=V_{u}^{\dagger} V_{d}
$$

We find that the following part in Eq. (25) induces FCNCs at tree level by the exchange of heavy neutral scalars $H, A$, with the strength controlled by the CKM matrix multiplied by quark masses:

$$
\begin{aligned}
-\mathcal{L}_{\text {yukawa }} \supset & \frac{\left(V m_{d}^{D} V^{\dagger}\right)_{i j}}{v \cos 2 \beta}\left(\bar{u}^{i} u^{j} H+i \bar{u}^{i} \gamma_{5} u^{j} A\right) \\
& +\frac{\left(V^{\dagger} m_{u}^{D} V\right)_{i j}}{v \cos 2 \beta}\left(\bar{d}^{i} d^{j} H-i \bar{d}^{i} \gamma_{5} d^{j} A\right) .
\end{aligned}
$$

Flavor violation is suppressed by off-diagonal components of the CKM matrix, and possibly by light quark masses, which is a characteristic property of the model.

Since the Yukawa couplings always appear in combination with the factor $1 / \cos 2 \beta$, hereafter we redefine the heavy scalar masses as ${ }^{6}$

$$
\begin{aligned}
\text { new } m_{H^{ \pm}}^{2} & \equiv m_{H^{ \pm}}^{2} \cos ^{2} 2 \beta, \quad \text { new } m_{H}^{2} \equiv m_{H}^{2} \cos ^{2} 2 \beta \\
\text { new } m_{A}^{2} & \equiv m_{A}^{2} \cos ^{2} 2 \beta
\end{aligned}
$$

As a reference, we present the absolute values of the flavor-violating part of the Yukawa couplings for $s_{f}=+1(f=u, c, t, d, s, b)$ :

$$
\begin{aligned}
& \text { For up - type }: \frac{\left|V m_{d}^{D} V^{\dagger}\right|}{v} \\
& \quad=\left(\begin{array}{ccc}
0.000037 & 0.000080 & 0.000059 \\
* & 0.00038 & 0.00068 \\
* & * & 0.017
\end{array}\right),
\end{aligned}
$$

\footnotetext{
${ }^{6}$ If we require $\tan \beta \sim m_{t} / m_{b}$ so that the top and bottom quark mass ratio is derived without fine-tuning, we have $\cos ^{2} 2 \beta \simeq 1$ and this redefinition becomes trivial.
}

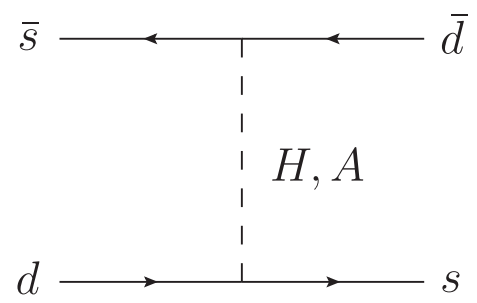

FIG. 1. Diagram for $\Delta S=2$ process arising from the exchange of a heavy neutral scalar particle $H, A$, which we call "flavor changing neutral Higgs (FCNH)". Another crossed diagram is omitted.

$$
\begin{aligned}
& \text { For down - type: } \frac{\left|V^{\dagger} m_{u}^{D} V\right|}{v} \\
& =\left(\begin{array}{ccc}
0.00032 & 0.0013 & 0.0060 \\
* & 0.0058 & 0.027 \\
* & * & 0.70
\end{array}\right) .
\end{aligned}
$$

The off-diagonal components in the above matrices indicate the strength of FCNCs mediated by the neutral scalars. Here, the CKM matrix components are obtained from the Wolfenstein parameters reported by the CKMfitter, which read [12],

$\lambda=0.22548, \quad A=0.810, \quad \bar{\rho}=0.145, \quad \bar{\eta}=0.343$.

We comment in passing that the Yukawa couplings for leptons and $H^{ \pm}, H$ and $A$ are obtained by a simple replacement: $d \rightarrow e, u \rightarrow \nu, M_{d} \rightarrow M_{e}, M_{u} \rightarrow M_{D}$ in Eq. (21), with $M_{D}$ being the neutrino Dirac mass involved in the seesaw mechanism. Due to our ignorance of the seesaw scale, we cannot predict the strength of the flavorviolating couplings for charged leptons.

\section{III. $\Delta F=2$ AMPLITUDES}

We give formulas for $\Delta F=2$ amplitudes to analyze flavor observables in the quark sector. In particular, mass differences for $K, B_{d}^{0}$ and $B_{s}^{0}$, and a $C P$ violating observable in kaon system are given. The effective Hamiltonian contributing to $\Delta S=2$ processes is given as follows,

$$
\begin{gathered}
\mathcal{H}_{\Delta S=2}^{\mathrm{FCNH}}=\frac{G_{F}}{\sqrt{2}}\left(C_{S} O_{S}+C_{P} O_{P}\right)+\text { H.c., } \\
C_{S}=-\frac{1}{m_{H}^{2}}\left[\sum_{k}^{u, c, t} \lambda_{k}^{s d}\left(m_{u}^{D}\right)^{k}\right]^{2}, \\
C_{P}=\frac{1}{m_{A}^{2}}\left[\sum_{k}^{u, c, t} \lambda_{k}^{s d}\left(m_{u}^{D}\right)^{k}\right]^{2}, \quad \lambda_{k}^{i j}=V_{k i}^{*} V_{k j}, \\
O_{S}=\bar{s} d \bar{s} d, \quad O_{P}=\bar{s} \gamma_{5} d \bar{s} \gamma_{5} d .
\end{gathered}
$$

Proper replacement of the indices in Eqs. (32)-(34) enables us to write the effective Hamiltonian in $\Delta B=2$ processes. The Hamiltonian in Eq. (32) represents the contribution of a tree-level diagram in Figure 1 arising from the exchange of 
a heavy neutral scalar particle $H, A$, which we denote "flavor changing neutral Higgs (FCNH)." When the heavy scalar particles are degenerate, one can obtain a further simplified Hamiltonian. Including the SM contribution, we can write,

$$
\begin{gathered}
\mathcal{H}_{\Delta S=2}=\mathcal{H}_{\Delta S=2}^{\mathrm{SM}}+\mathcal{H}_{\Delta S=2}^{\mathrm{FCNH},} \\
\mathcal{H}_{\Delta S=2}^{\mathrm{SM}}=C_{1}^{\mathrm{VLL}} Q_{1}^{\mathrm{VLL}}+\text { H.c., } \\
\mathcal{H}_{\Delta S=2}^{\mathrm{FCNH}}=\sum_{i=1}^{2} C_{i}^{\mathrm{LR}} Q_{i}^{\mathrm{LR}}+\text { H.c. },
\end{gathered}
$$

where we follow the notation of Ref. [13]. In Eq. (37), we do not include operators, $Q_{1}^{\mathrm{SLL}}, Q_{2}^{\mathrm{SLL}}$ and other chiralityflipped ones given in Ref. [13], since they do not arise from the FCNH diagram. Furthermore, $Q_{1}^{\mathrm{SLL}}$ and $Q_{2}^{\mathrm{SLL}}$ are decoupled from the mixing with the other operators so that we omit these contributions. The Wilson coefficients and the operators in Eqs. (36), (37) are given as,

$$
\begin{gathered}
C_{1}^{\mathrm{VLL}}=\frac{G_{F}^{2} M_{W}^{2}}{(4 \pi)^{2}} 4 \tilde{S}, \quad C_{1}^{\mathrm{LR}}\left(\mu_{H}\right)=0, \\
C_{2}^{\mathrm{LR}}\left(\mu_{H}\right)=-\frac{2 \sqrt{2} G_{F}}{m_{H}^{2}}\left[\sum_{k}^{u, c, t} \lambda_{k}^{s d}\left(m_{u}^{D}\right)^{k}\right]^{2}, \\
Q_{1}^{\mathrm{VLL}}=\bar{s}^{\alpha} \gamma^{\mu} P_{L} d^{\alpha} \bar{s}^{\beta} \gamma_{\mu} P_{L} d^{\beta}, \\
Q_{1}^{\mathrm{LR}}=\bar{s}^{\alpha} \gamma_{\mu} P_{L} d^{\alpha} \bar{s}^{\beta} \gamma^{\mu} P_{R} d^{\beta}, \\
Q_{2}^{\mathrm{LR}}=\bar{s}^{\alpha} P_{L} d^{\alpha} \bar{s}^{\beta} P_{R} d^{\beta},
\end{gathered}
$$

where $P_{R(L)}=\left(1 \pm \gamma_{5}\right) / 2$ denotes chirality projection operators while $\alpha$ and $\beta$ represent color indices. In Eq. (38), $C_{1}^{\mathrm{VLL}}$ stands for the contribution within the SM, and the Inami-Lim function [14] is given as,

$$
\begin{gathered}
\tilde{S}=\eta_{1}\left(\lambda_{c}^{s d}\right)^{2} S\left(x_{c}\right)+\eta_{2}\left(\lambda_{t}^{s d}\right)^{2} S\left(x_{t}\right)+2 \eta_{3} \lambda_{c}^{s d} \lambda_{t}^{s d} S\left(x_{c}, x_{t}\right), \\
x_{i}=\frac{m_{i}^{2}}{M_{W}^{2}} \quad(i=c, t), \\
S\left(x_{i}, x_{j}\right)=x_{i} x_{j}\left[\frac { 1 } { x _ { i } - x _ { j } } \left(\left(\frac{1}{4}-\frac{3}{2} \frac{1}{x_{i}-1}-\frac{3}{4} \frac{1}{\left(x_{i}-1\right)^{2}}\right) \ln x_{i}\right.\right. \\
\left.-\left(\frac{1}{4}-\frac{3}{2} \frac{1}{x_{j}-1}-\frac{3}{4} \frac{1}{\left(x_{j}-1\right)^{2}}\right) \ln x_{j}\right) \\
\left.-\frac{3}{4} \frac{1}{\left(x_{i}-1\right)\left(x_{j}-1\right)}\right] \\
S\left(x_{i}\right)=\frac{4 x_{i}-11 x_{i}^{2}+x_{i}^{3}}{4\left(x_{i}-1\right)^{2}}+\frac{3}{2}\left(\frac{x_{i}}{x_{i}-1}\right)^{3} \ln x_{i}
\end{gathered}
$$

where NLO QCD correction factors within the SM, $\left(\eta_{1}, \eta_{2}, \eta_{3}\right)$, have been calculated in Ref. [15]. To be precise, one should multiply Eq. (40) by an overall factor which accounts renormalization scale of lattice QCD calculation.

As for the $\Delta B=2$ processes, we only take account of the contribution of internal top quarks, and the corresponding NLO QCD correction is obtained through the method in Ref. [13]. The formulae for an anomalous dimension matrix including two-loop contribution are given in Ref. [16]. As remarked in the literature [13], this renormalization group effect drastically enhances $C_{2}^{\mathrm{LR}}$ while it does not significantly change $C_{1}^{\mathrm{LR}}$. In our analysis, new world averages of the QCD scale obtained by PDG [17] are used.

The matrix elements of the $\Delta F=2$ transition are parametrized as,

$$
\begin{aligned}
& \left\langle\bar{K}^{0}\left|Q_{1}^{\mathrm{VLL}}(\mu)\right| K^{0}\right\rangle=\frac{1}{3} M_{K} f_{K}^{2} B_{1}^{\mathrm{VLL}}(\mu), \\
& \left\langle\bar{K}^{0}\left|Q_{1}^{\mathrm{LR}}(\mu)\right| K^{0}\right\rangle=-\frac{1}{6} M_{K} f_{K}^{2} B_{1}^{\mathrm{LR}}(\mu)\left(\frac{M_{K}}{m_{s}(\mu)+m_{d}(\mu)}\right)^{2},
\end{aligned}
$$

$$
\left\langle\bar{K}^{0}\left|Q_{2}^{\mathrm{LR}}(\mu)\right| K^{0}\right\rangle=\frac{1}{4} M_{K} f_{K}^{2} B_{2}^{\mathrm{LR}}(\mu)\left(\frac{M_{K}}{m_{s}(\mu)+m_{d}(\mu)}\right)^{2},
$$

$\left\langle\bar{B}_{q}^{0}\left|Q_{1}^{\mathrm{VLL}}(\mu)\right| B_{q}^{0}\right\rangle=\frac{1}{3} M_{B_{q}} f_{B_{q}}^{2} B_{1}^{q \mathrm{VLL}}(\mu)$,

$\left\langle\bar{B}_{q}^{0}\left|Q_{1}^{\mathrm{LR}}(\mu)\right| B_{q}^{0}\right\rangle=-\frac{1}{6} M_{B_{q}} f_{B_{q}}^{2} B_{1}^{q \mathrm{LR}}(\mu)\left(\frac{M_{B_{q}}}{m_{b}(\mu)+m_{q}(\mu)}\right)^{2}$,

$$
\left\langle\bar{B}_{q}^{0}\left|Q_{2}^{\mathrm{LR}}(\mu)\right| B_{q}^{0}\right\rangle=\frac{1}{4} M_{B_{q}} f_{B_{q}}^{2} B_{2}^{q \mathrm{LR}}(\mu)\left(\frac{M_{B_{q}}}{m_{b}(\mu)+m_{q}(\mu)}\right)^{2} .
$$

where $q=d, s$. In this normalization, kaon decay constant is given as $f_{K}=156.1 \mathrm{MeV}$. The matrix elements in Eqs. (43)-(48) are written in terms of bag parameters, which represent the deviation from vacuum saturation approximation. For these parameter, we use the data which are calculated by the ETM collaboration $[18,19]$. Their results are obtained in M S scheme, and extracted from the result in the supersymmetric basis. The correspondence between bag parameters in the operator basis in Eq. (39) and ones in the supersymmetric basis is given in Ref. [13].

The mass differences of neutral meson system are obtained as follows,

$$
\Delta M_{K}=2 \operatorname{Re} M_{12}, \quad \Delta M_{B_{q}}=2\left|M_{12}^{q}\right|,
$$


TABLE II. Input data used in the analysis. The third column corresponds to references on which the data are based. For $K_{L}-$ $K_{S}$ mass difference, the fitting result w/CPT assumption is given. $\operatorname{Re} \epsilon$ is extracted from asymmetry of semileptonic decay rates given by PDG.

\begin{tabular}{lcc}
\hline \hline$\eta_{1}$ & $1.32_{-0.23}^{+0.21}$ & {$[15]$} \\
$\eta_{2}$ & $0.57_{-0.01}^{+0.00}$ & {$[15]$} \\
$\eta_{3}$ & $0.47_{-0.04}^{+0.03}$ & {$[15]$} \\
$\Lambda_{\overline{\mathrm{MS}}}^{(6)}$ & $(87 \pm 7) \mathrm{MeV}$ & {$[17]$} \\
$\Lambda_{\overline{\mathrm{MS}}}^{(5)}$ & $(210 \pm 15) \mathrm{MeV}$ & {$[17]$} \\
$\Lambda_{\overline{\mathrm{MS}}}^{(4)}$ & $(291 \pm 19) \mathrm{MeV}$ & {$[17]$} \\
$\operatorname{Re} \epsilon$ & $(1.66 \pm 0.03) \times 10^{-3}$ & {$[17]$} \\
$\Delta M_{K}$ & $3.484 \pm 0.006\left[10^{-12} \mathrm{MeV}\right]$ & {$[17]$} \\
$\Delta M_{B_{d}}$ & $0.5064 \pm 0.0019\left[\mathrm{ps}{ }^{-1}\right]$ & {$[20]$} \\
$\Delta M_{B_{s}}$ & $17.757 \pm 0.0021\left[\mathrm{ps}{ }^{-1}\right]$ & {$[20]$} \\
$\sin 2 \beta_{\mathrm{eff}}$ & $0.691 \pm 0.017$ & {$[20]$} \\
$B_{1}^{\mathrm{VLL}}(3 \mathrm{GeV})$ & $0.506 \pm 0.017$ & {$[18]$} \\
$B_{1}^{\mathrm{LR}}(3 \mathrm{GeV})$ & $0.49 \pm 0.04$ & {$[18]$} \\
$B_{2}^{\mathrm{LR}}(3 \mathrm{GeV})$ & $0.78 \pm 0.05$ & {$[18]$} \\
$f_{B_{d}} \sqrt{B_{1}^{d \mathrm{VLL}}}\left(m_{b}\right)$ & $174 \pm 8 \mathrm{MeV}$ & {$[19]$} \\
$f_{B_{d}} \sqrt{B_{1}^{d \mathrm{LR}}}\left(m_{b}\right)$ & $229 \pm 14 \mathrm{MeV}$ & {$[19]$} \\
$f_{B_{d}} \sqrt{B_{2}^{d \mathrm{LR}}}\left(m_{b}\right)$ & $185 \pm 9 \mathrm{MeV}$ & {$[19]$} \\
$f_{B_{s}} \sqrt{B_{1}^{s \mathrm{VLL}}}\left(m_{b}\right)$ & $211 \pm 8 \mathrm{MeV}$ & {$[19]$} \\
$f_{B_{s}} \sqrt{B_{1}^{s \mathrm{LR}}}\left(m_{b}\right)$ & $285 \pm 14 \mathrm{MeV}$ & {$[19]$} \\
$f_{B_{s}} \sqrt{B_{2}^{s \mathrm{LR}}}\left(m_{b}\right)$ & $220 \pm 9 \mathrm{MeV}$ & {$[19]$} \\
\hline \hline & &
\end{tabular}

$M_{12}=M_{12}^{\mathrm{SM}}+M_{12}^{\mathrm{FCNH}}, \quad M_{12}^{q}=M_{12}^{q \mathrm{SM}}+M_{12}^{q \mathrm{FCNH}}$,

where $M_{12}^{(q)}$ is divided into the SM part and the new physics part,

$M_{12}^{\mathrm{SM}}=\left\langle\bar{K}^{0}\left|\mathcal{H}_{\Delta S=2}^{\mathrm{SM}}\right| K^{0}\right\rangle^{*}, \quad M_{12}^{\mathrm{FCNH}}=\left\langle\bar{K}^{0}\left|\mathcal{H}_{\Delta S=2}^{\mathrm{FCNH}}\right| K^{0}\right\rangle^{*}$,

$M_{12}^{q \mathrm{SM}}=\left\langle\bar{B}_{q}^{0}\left|\mathcal{H}_{\Delta B=2}^{\mathrm{SM}}\right| B_{q}^{0}\right\rangle^{*}, \quad M_{12}^{q \mathrm{FCNH}}=\left\langle\bar{B}_{q}^{0}\left|\mathcal{H}_{\Delta B=2}^{\mathrm{FCNH}}\right| B_{q}^{0}\right\rangle^{*}$.

Moreover, indirect $C P$ violation in kaon system is characterized by

$$
\epsilon=\frac{e^{\frac{i \pi}{4}}}{\sqrt{2}} \frac{\operatorname{Im} M_{12}}{\Delta M_{K}}
$$

\section{CURRENT BOUND ON THE MODEL}

In this section, we obtain the bound on mass of heavy Higgs through flavor observables. First, $\sin 2 \beta_{\text {eff }}$, which represents $C P$ violation of interference in $B_{d}^{0}-\bar{B}_{d}^{0}$ mixing and $B_{d}^{0} \rightarrow J / \psi K_{S}$, is analyzed. As discussed later, the bounds on Higgs mass are determined by p-values of CKM fitting.

In Table II, input data which appear in the numerical analysis are summarized.

\section{A. $\sin 2 \beta_{\text {eff }}$ measured in $B_{d}^{0} \rightarrow J / \psi K_{S}$ decay}

Throughout this paper, we assume that direct $C P$ violation in $B_{d}^{0} \rightarrow J / \psi K_{S}$ decay is negligible. With this approximation, the time-dependent $C P$ asymmetry in $B_{d}^{0} \rightarrow J / \psi K_{S}$ decay is given as follows,

$$
\begin{gathered}
A_{C P}(t) \simeq S_{J / \psi K_{S}} \sin \left(\Delta M_{B_{d}} t\right), \\
S_{J / \psi K_{S}} \simeq \operatorname{Im}\left(\frac{q}{p} \frac{\bar{A}_{J / \psi K_{S}}}{A_{J / \psi K_{S}}}\right),
\end{gathered}
$$

where $q / p$ is a mixing parameter [21] in $B_{d}^{0}$ system while $A_{J / \psi K_{S}}\left(\bar{A}_{J / \psi K_{S}}\right)$ represents decay amplitude of $B_{d}^{0}\left(\bar{B}_{d}^{0}\right) \rightarrow J / \psi K_{S}$. In the semialigned $2 \mathrm{HDM}$, the decay amplitude in Eq. (55) does not deviate from the SM prediction, since a diagram of the charged scalar exchange gives rise to minor modification due to smallness of Yukawa couplings. We do not take account of such negligible contribution for the decay amplitude. Furthermore, the correction coming from penguin pollution in the SM is also small [22] because of the suppression for the CKM and the loop factor, and hence we ignore this effect. Meanwhile, the mixing parameter for $B_{d}^{0}$ system, $q / p$, and one for kaon system are modified due to the diagrams for FCNH exchange. Hence, the parameter in Eq. (55) is given as [23,24],

$$
\begin{gathered}
\sin 2 \beta_{\mathrm{eff}}=S_{J / \psi K_{S}}=\sin \left[2 \beta+\arg \left(1+\frac{M_{12}^{d \mathrm{FCNH}}}{M_{12}^{d \mathrm{SM}}}\right)\right. \\
\left.-\arg \left(1+\frac{M_{12}^{\mathrm{FCNH}}}{M_{12}^{\mathrm{SM}}}\right)\right], \\
\beta=\arg \left(-\frac{V_{c d} V_{c b}^{*}}{V_{t d} V_{t b}^{*}}\right),
\end{gathered}
$$

where the definitions of $M_{12}^{(d) \mathrm{SM}}$ and $M_{12}^{(d) \mathrm{FCNC}}$ are given in Eqs. (51), (52). Thus, the experimental observable deviates from $\sin 2 \beta$ due to modification of the mixing parameters.

In Fig. 2 , in order to illustrate the FCNH mass dependence of $\sin 2 \beta_{\text {eff }}$, we plot,

$$
\arg \left(1+\frac{M_{12}^{d \mathrm{FCNH}}}{M_{12}^{d S \mathrm{~S}}}\right),
$$

where for the CKM matrix components, we used the values in Eq. (31). The numerical behavior of the argument in Eq. (58) can be understood in the following way: If one 


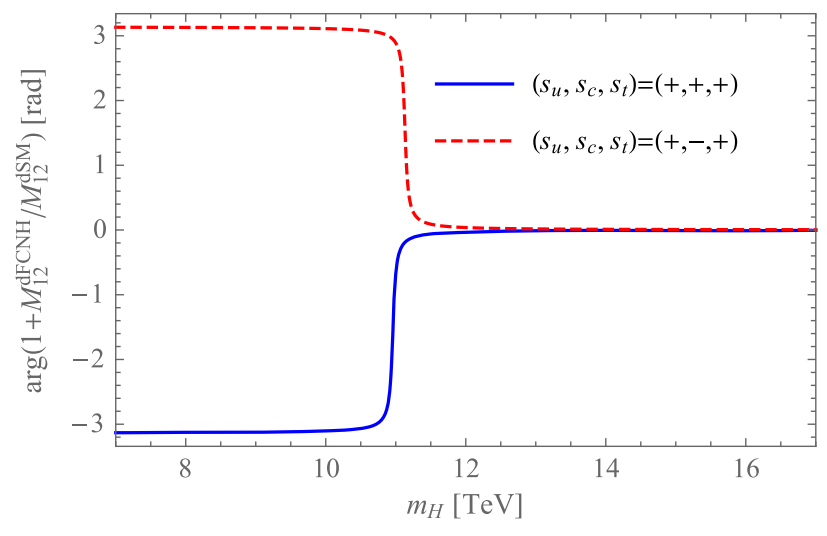

FIG. 2. Argument of $1+M_{12}^{d \mathrm{FCNH}} / M_{12}^{d \mathrm{SM}}$.

neglects masses of up and charm quarks, a phase for $M_{12}^{d \mathrm{FCNH}}$ is determined as $-\left(V^{\dagger} m_{u}^{D} V\right)_{b d}^{2} \propto-\left(V_{t b}^{*} V_{t d}\right)^{2}$. This factor is the same as the box diagram in the SM up to its sign. Therefore, $M_{12}^{d \mathrm{FCNH}} / M_{12}^{d \mathrm{SM}}$ is a negative real number, approximately. Owing to this fact, we find

$$
\left\{\begin{array}{l}
\arg \left(1+M_{12}^{d \mathrm{FCNH}} / M_{12}^{d \mathrm{SM}}\right)=0, \quad\left(-1<M_{12}^{d \mathrm{FCNH}} / M_{12}^{d \mathrm{SM}}<0\right) \\
\arg \left(1+M_{12}^{d \mathrm{FCNH}} / M_{12}^{d \mathrm{SM}}\right)= \pm \pi, \quad\left(M_{12}^{d \mathrm{FCNH}} / M_{12}^{d \mathrm{SM}}<-1\right)
\end{array}\right.
$$

where for $M_{12}^{d \mathrm{FCNH}} / M_{12}^{d \mathrm{SM}}<-1$, sign of the argument depends on sign of tiny imaginary part in the argument. The relations in Eq. (59) indicate that the argument in Eq. (58) vanishes if $m_{H}$ is sufficiently large. From Fig. 2, we find that the argument in Eq. (59) does not affect $\sin 2 \beta_{\text {eff }}$ for $m_{H} \geq 12 \mathrm{TeV}$. Note that in fact, charm quark mass slightly contributes to the above quantity so that the exact behavior in Fig. 2 is not identical to Eq. (59).

We should also note that quark mass signs in Eq. (24) give rise to numerical difference in the argument in Eq. (58). However, choice of $s_{u}= \pm 1$ yields a minor difference since up quark mass is negligible. Thus, the two representative cases, $\left(s_{u}, s_{c}, s_{t}\right)=(+,+,+)$ and

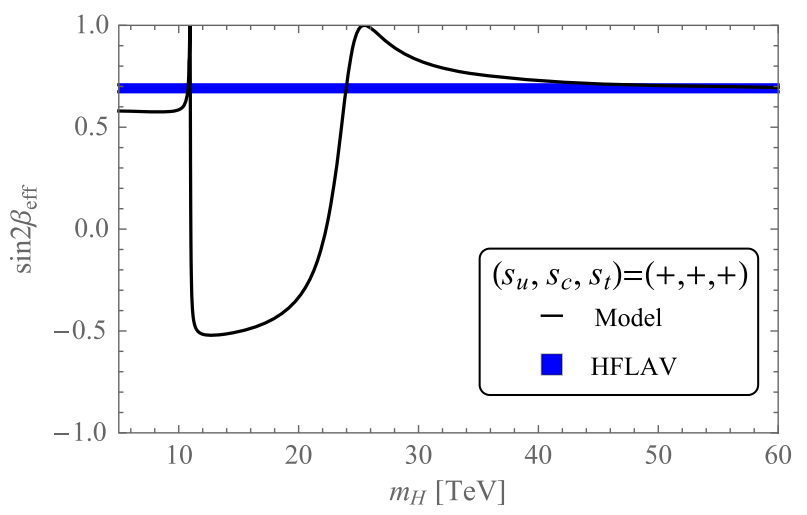

(a) $\left(s_{u}, s_{c}, s_{t}\right)=(+,-,+)$ are sufficient since the relative sign for $s_{c}$ and $s_{t}$ almost determines the coupling for $\Delta F=2$ processes in Eq. (38).

In Fig. 3, we show the prediction for $\sin 2 \beta_{\text {eff }}$ with $\left(s_{u}, s_{c}, s_{t}\right)=(+,+,+)$ and $\left(s_{u}, s_{c}, s_{t}\right)=(+,-,+)$. In the plot, one can find that $\sin 2 \beta_{\text {eff }}$ shows nontrivial dependence on Higgs mass since both $K$ and $B_{d}^{0}$ systems affect the observable. For $m_{H} \gg 50 \mathrm{TeV}$, the neutral Higgs decouples from $\sin 2 \beta_{\text {eff }}$ so that it becomes identical to the SM prediction asymptotically.

\section{B. Bound on $m_{H}$ from CKM fitting}

Now that we have obtained the formulae for flavorviolating observables, we derive bounds on FCNH mass.

It should be noted that in the presence of $\mathrm{FCNH}$, the values of CKM matrix components are altered from those found in the literature, since the $\mathrm{FCNH}$ exchange process modifies the theoretical formulas for $\Delta M_{B_{d}}, \Delta M_{B_{s}}$, Re $\epsilon$ and $\sin 2 \beta_{\text {eff }}$. In order to derive the bounds, we utilize $\mathrm{p}$-values in the CKM fitting for flavor-violating observables. The analysis is performed in the following way: We carry out $\chi^{2}$ fittings with fixed $m_{H}$. For each value of $m_{H}$, p-values are given to specify a disfavored range of Higgs mass. Given the stringent experimental constraint on $\operatorname{Re} \epsilon$, the FCNH must be sufficiently heavy. Thus, $m_{H} \geq 20 \mathrm{TeV}$ is considered in the fittings.

For completeness, we show the Wolfenstein parametrization of the CKM matrix [25],

$V=\left(\begin{array}{ccc}1-\lambda^{2} / 2 & \lambda & A \lambda^{3}(\rho-i \eta) \\ -\lambda & 1-\lambda^{2} / 2 & A \lambda^{2} \\ A \lambda^{3}(1-\rho-i \eta) & -A \lambda^{2} & 1\end{array}\right)$

where terms of $\mathcal{O}\left(\lambda^{4}\right)$ are ignored. In Eq. $(60),(\rho, \eta)$ are redefined in terms of phase convention independent parameters, $(\bar{\rho}, \bar{\eta})[26]$,

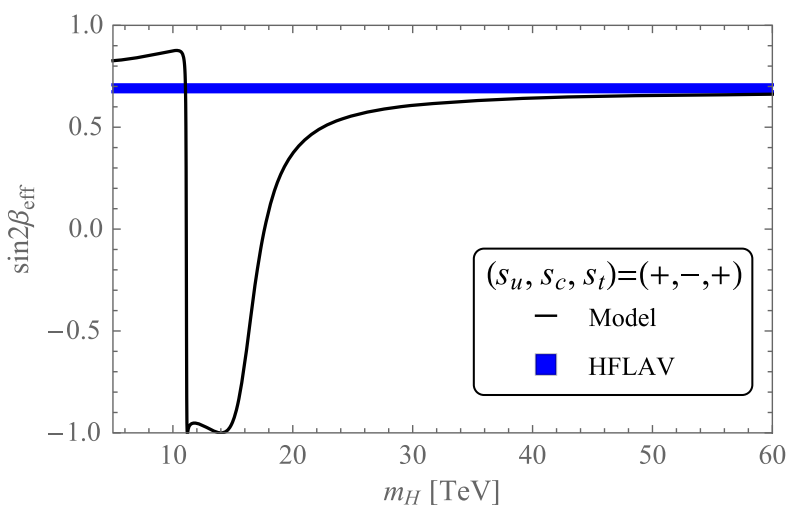

(b)

FIG. 3. Model prediction for $\sin 2 \beta_{\text {eff }}$ with (a) $\left(s_{u}, s_{c}, s_{t}\right)=(+,+,+)$ and (b) $\left(s_{u}, s_{c}, s_{t}\right)=(+,-,+)$. 


$$
\rho+i \eta=\frac{(\bar{\rho}+i \bar{\eta}) \sqrt{1-A^{2} \lambda^{4}}}{\sqrt{1-\lambda^{2}}\left[1-A^{2} \lambda^{4}(\bar{\rho}+i \bar{\eta})\right]} .
$$

In addition, angles which constitute the unitarity triangle are defined as,

$$
\begin{aligned}
& \alpha=\arg \left(-\frac{V_{t d} V_{t b}^{*}}{V_{u d} V_{u b}^{*}}\right), \\
& \gamma=\arg \left(-\frac{V_{u d} V_{u b}^{*}}{V_{c d} V_{c b}^{*}}\right),
\end{aligned}
$$

where the other angle, $\beta$, is given in Eq. (57). We note that $(\alpha, \beta, \gamma)$ can be written in terms of the Wolfenstein parameters, $(\lambda, A, \bar{\rho}, \bar{\eta})$.

In carrying out the analysis, the following facts are considered:

(i) Measurements of $\left|V_{u d}\right|,\left|V_{u s}\right|,\left|V_{u b}\right|,\left|V_{c d}\right|,\left|V_{c s}\right|$ and $\left|V_{c b}\right|$ are unchanged in the presence of FCNH. This is because these absolute values are determined through semileptonic decays, while the model predicts minor correction for semileptonic processes.

(ii) Angle $\alpha$, which is measured in time-dependent processes for $B \rightarrow \pi \pi, B \rightarrow \pi \rho$ and $B \rightarrow \rho \rho$ decays, is altered by $\mathrm{FCNH}$, since these observables are sensitive to $B_{d}^{0}-\bar{B}_{d}^{0}$ mixing. However, as stated in the previous subsection, the FCNH contribution to $B_{d}^{0}-\bar{B}_{d}^{0}$ mixing does not affect $q / p$ for $m_{H} \geq$ $12 \mathrm{TeV}$ so that we decouple this effect.

(iii) Measurement of angle $\gamma$ is carried out in $B^{ \pm} \rightarrow D K^{ \pm}$ and $B^{0} \rightarrow D^{(*) \pm} \pi^{\mp}$ decays. These tree-level processes are not significantly modified by the FCNH exchange.

In the fitting, we include uncertainties of the Wolfenstein parameters [12], the bag parameters [18,19] and NLO QCD correction factors for the $\Delta S=2$ process denoted by $\eta_{1}, \eta_{2}$ and $\eta_{3}$ [15]. As for the $\Delta B=2$ processes, uncertainty in short-distance QCD correction factors is not considered because they are more precisely determined than those for the $\Delta S=2$ process. We use the central value of this QCD correction calculated through the method of Ref. [13].

In the CKM fitting, a statistic,

$$
\begin{aligned}
\left(\chi^{2}\right)_{\text {fixed } m_{H}}= & \sum_{i, j} \frac{\left(\left|V_{i j}\right|_{\mathrm{Th}}-\left|V_{i j}\right|_{\operatorname{Exp}}\right)^{2}}{\left(\left.\sigma_{\mid V_{i j}}\right|_{\operatorname{Exp}} ^{2}\right.} \\
& +\sum_{k=\alpha, \gamma, \sin 2 \beta_{\text {eff }}} \frac{\left(k_{\mathrm{Th}}-k_{\operatorname{Exp}}\right)^{2}}{\left(\sigma_{k}\right)_{\operatorname{Exp}}^{2}} \\
& +\sum_{l=d, s} \frac{\left(\Delta M_{B_{l} \mathrm{Th}}-\Delta M_{B_{l} \operatorname{Exp}}\right)^{2}}{\left(\sigma_{\Delta M_{B_{l}}}\right)_{\mathrm{Th}}^{2}+\left(\sigma_{\Delta M_{B_{l}}}\right)_{\operatorname{Exp}}^{2}} \\
& +\frac{\left(\operatorname{Re} \epsilon_{\mathrm{Th}}-\operatorname{Re} \epsilon_{\operatorname{Exp}}\right)^{2}}{\left(\sigma_{\operatorname{Re} \epsilon}\right)_{\mathrm{Th}}^{2}+\left(\sigma_{\operatorname{Re} \epsilon}\right)_{\operatorname{Exp}}^{2}}
\end{aligned}
$$

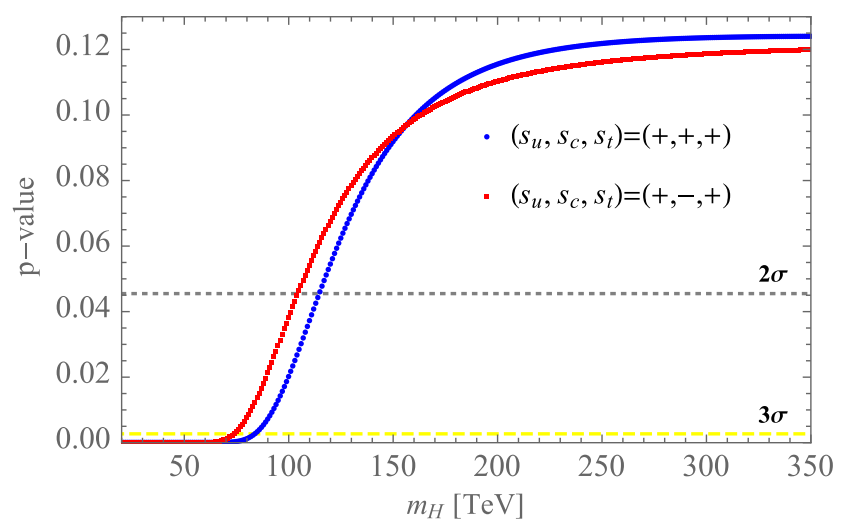

FIG. 4. P-value obtained in CKM fitting. Blue dots and red rectangles represent the cases of $\left(s_{u}, s_{c}, s_{t}\right)=(+, \pm,+)$. A gray dotted line and a yellow dashed line stand for $(100-95.4) \%$ and $(100-99.7) \%$, which correspond to p-values of $2 \sigma$ and $3 \sigma$ confidence levels (CLs), respectively.

is minimized with $(i, j)=(u, d),(u, s),(c, d),(c, s)$, $(c, b),(u, b)$. Experimental data on the absolute values of the CKM matrix elements and $\alpha, \gamma$ are provided by PDG as,

$$
\begin{aligned}
\left|V_{u d}\right|_{\operatorname{Exp}} & =0.97417 \pm 0.00021, \\
\left|V_{u s}\right|_{\operatorname{Exp}} & =0.2248 \pm 0.0006, \\
\left|V_{c d}\right|_{\operatorname{Exp}} & =0.220 \pm 0.005, \\
\left|V_{c s}\right|_{\operatorname{Exp}} & =0.995 \pm 0.016, \\
\left|V_{c b}\right|_{\operatorname{Exp}} & =(40.5 \pm 1.5) \times 10^{-3}, \\
\left|V_{u b}\right|_{\operatorname{Exp}} & =(4.09 \pm 0.39) \times 10^{-3}, \\
\alpha_{\operatorname{Exp}} & =(87.6 \pm 3.4)^{\circ}, \\
\gamma_{\operatorname{Exp}} & =(73.2 \pm 6.7)^{\circ},
\end{aligned}
$$

where we have taken averages of errors for $\alpha_{\operatorname{Exp}}$ and $\gamma_{\operatorname{Exp}}$, which are originally given as asymmetric forms. The data of $\left(\sin 2 \beta_{\text {eff }}\right)_{\operatorname{Exp}},(\operatorname{Re} \epsilon)_{\operatorname{Exp}},\left(\Delta M_{B_{d}}\right)_{\operatorname{Exp}},\left(\Delta M_{B_{s}}\right)_{\operatorname{Exp}}$ are extracted from Table II. In the r.h.s of Eq. (64), note that Higgs mass is fixed and the Wolfenstein parameters are adjustable to minimize $\left(\chi^{2}\right)_{\text {fixed } m_{H}}$. Furthermore, in Eq. (64), the theoretical errors for the bag parameters in $\Delta M_{B_{d}}, \Delta M_{B_{s}}$ are added to experimental ones in quadrature while for $\operatorname{Re} \epsilon$, the errors of perturbative QCD factors $\left(\eta_{1}, \eta_{2}, \eta_{3}\right)$ are also accounted. Note that our fitting analysis is different from the one performed by the CKMfitter group [12], which is based on $R$ fit [27], another frequentist approach to include theoretical uncertainty.

In Fig. 4, p-values obtained for $20 \mathrm{TeV} \leq m_{H} \leq$ $350 \mathrm{TeV}$ are presented. One observes a difference between $s_{u} s_{c}=+1$ and $s_{u} s_{c}=-1$. From Fig. 4, we derive lower bounds on the FCNH mass in which p-values are disfavored by $3 \sigma$ and $5 \sigma$. For the two cases of sign choice, the bounds are 


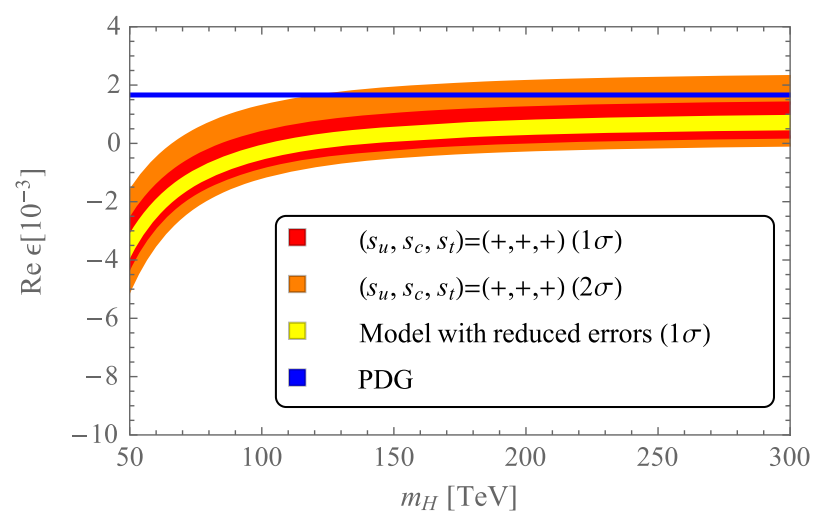

(a)

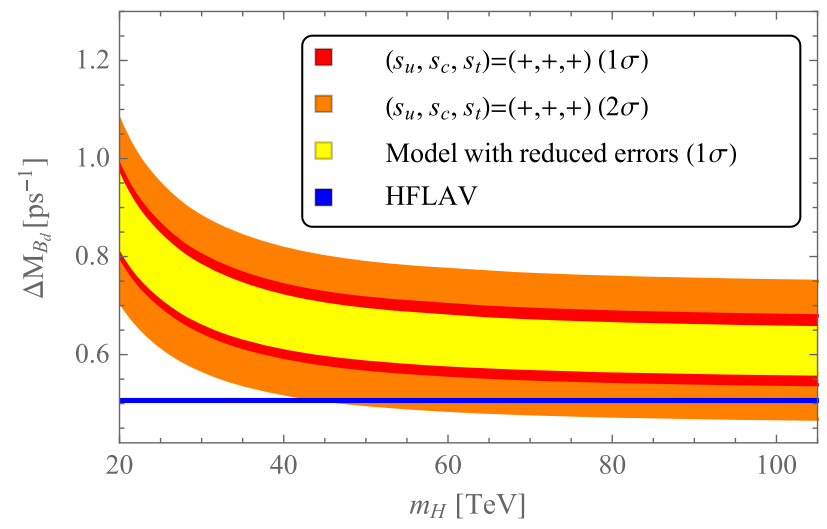

(c)

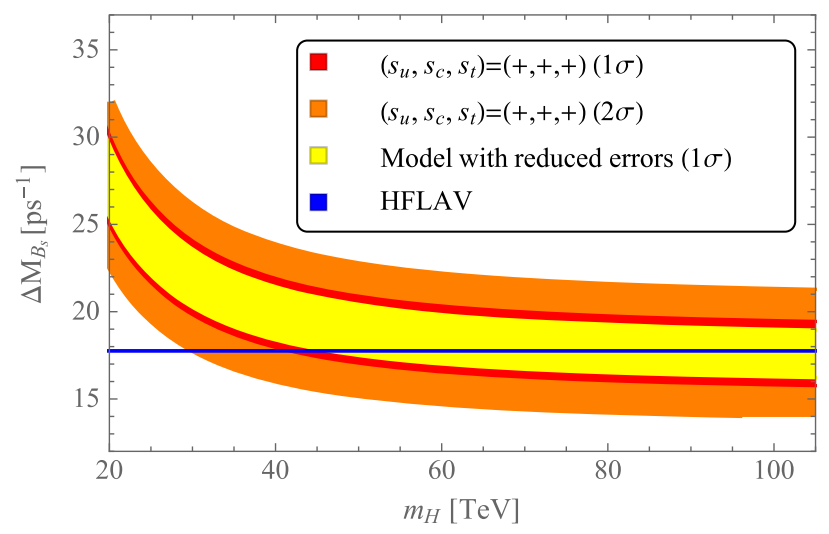

(e)

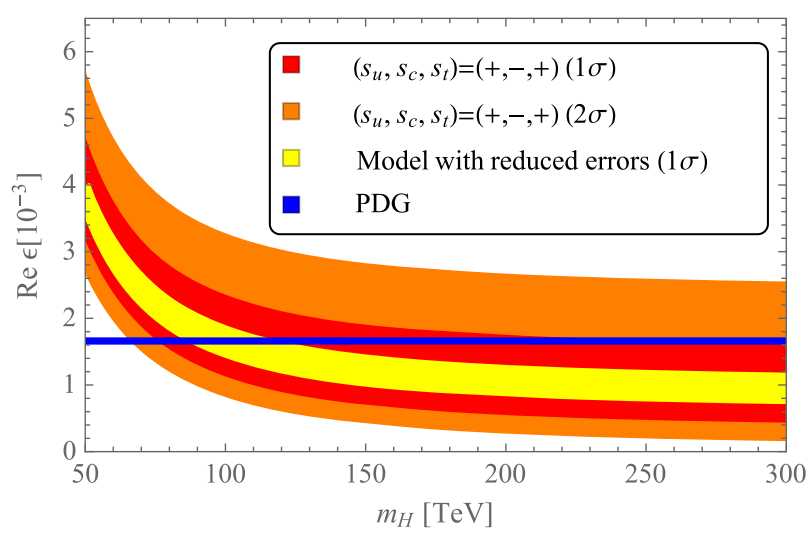

(b)

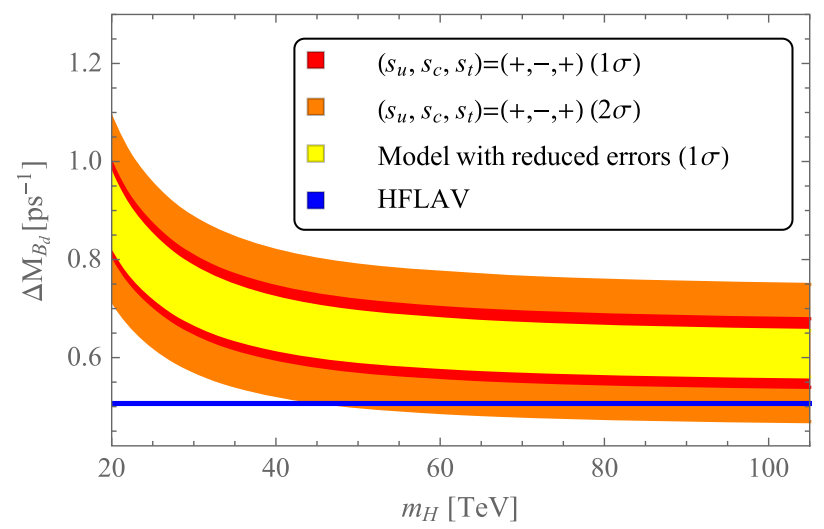

(d)

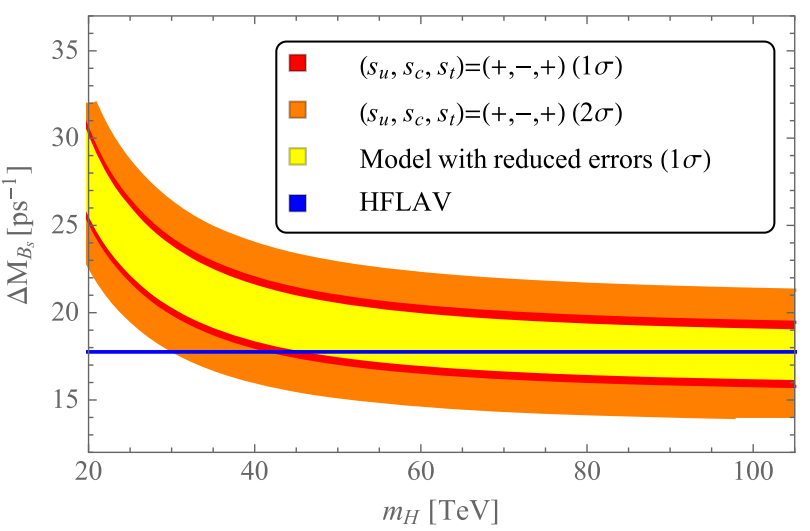

(f)

FIG. 5. Model predictions for Re $\epsilon$ and $\Delta M_{B_{d}}, \Delta M_{B_{s}}$ in Case I. In these plots, the Wolfenstein parameters used are estimated through observables which are not affected by FCNH. Red (orange) bands represent the model predictions in $1 \sigma(2 \sigma)$ CL. Yellow bands stand for the model predictions in which the errors of decay constant or bag parameters are three times as small as the one given by the ETM collaboration. For comparison, the experimental data of PDG and HFLAV are shown.

For $\left(s_{u}, s_{c}, s_{t}\right)=(+,+,+)$,

$m_{H}>84 \mathrm{TeV}(3 \sigma), \quad m_{H}>64 \mathrm{TeV}(5 \sigma)$,

For $\left(s_{u}, s_{c}, s_{t}\right)=(+,-,+)$,

$m_{H}>75 \mathrm{TeV}(3 \sigma), \quad m_{H}>56 \mathrm{TeV}(5 \sigma)$.

\section{PREDICTION FOR Re $\epsilon, \Delta M_{B_{d}}$ AND $\Delta M_{B_{s}}$}

In this section, we present the prediction for observables to illustrate the pattern of deviation in the model. For this purpose, the Wolfenstein parameters are estimated from observables which are not affected by FCNH. Hence, we consider the following statistic, 


$$
\chi^{2}=\sum_{i, j} \frac{\left(\left|V_{i j}\right|_{\mathrm{Th}}-\left|V_{i j}\right|_{\operatorname{Exp}}\right)^{2}}{\left(\sigma_{\left|V_{i j}\right|}\right)_{\operatorname{Exp}}^{2}}+\frac{\left(\gamma_{\mathrm{Th}}-\gamma_{\operatorname{Exp}}\right)^{2}}{\left(\sigma_{\gamma}\right)_{\operatorname{Exp}}^{2}} .
$$

Through the minimization of Eq. (67), one can extract $(\lambda, A, \bar{\rho}, \bar{\eta})$.

The Belle II experiment announces that the expected integrated luminosity is $50 \mathrm{ab}^{-1}$ in five years of running. Motivated by this, we consider Case I and Case II described below.

(i) Case I:

The errors of $\left|V_{u b}\right|$ and $\gamma$ are reduced by $1 / 3$ and $1 / 7$, respectively, without changing their central values. The errors and central values of the other quantities, including $\left|V_{c b}\right|$, remain the same. Under this circumstance, the parameters are estimated as

$$
\begin{aligned}
& \lambda=0.22547 \pm 0.00050, \quad A=0.797 \pm 0.030, \\
& \bar{\rho}=0.1262 \pm 0.093, \quad \bar{\eta}=0.418 \pm 0.021
\end{aligned}
$$

where a correlation matrix for $(\lambda, A, \bar{\rho}, \bar{\eta})$ is

$$
\left(\begin{array}{cccc}
1 & -0.12 & -0.032 & -0.048 \\
* & 1 & -0.50 & -0.74 \\
* & * & 1 & 0.59 \\
* & * & * & 1
\end{array}\right) .
$$

On the basis of the Wolfenstein parameters in Eq. (70), the prediction for $\left(\operatorname{Re} \epsilon, \Delta M_{B_{d}}, \Delta M_{B_{s}}\right)$ is given in Fig. 5. We also present the result for the case in which errors of bag parameters and decay constants are three times as small as the ones calculated by the ETM collaboration.

(ii) Case II:

Case II is an ideal situation in which the errors of $\left|V_{c b}\right|,\left|V_{u b}\right|$ and $\gamma$ are reduced by $1 / 7$ (the other quantities remain the same). In this case, the Wolfenstein parameters and their correlation matrix are,

$$
\begin{gathered}
\lambda=0.22547 \pm 0.00050, \quad A=0.7967 \pm 0.0055, \\
\bar{\rho}=0.1262 \pm 0.071, \quad \bar{\eta}=0.4180 \pm 0.0065, \\
\left(\begin{array}{ccccc}
1 & -0.65 & -0.042 & -0.15 \\
* & 1 & -0.044 & -0.16 \\
* & * & 1 & -0.063 \\
* & * & * & 1
\end{array}\right) .
\end{gathered}
$$

For Case II, the prediction for $\left(\operatorname{Re} \epsilon, \Delta M_{B_{d}}, \Delta M_{B_{s}}\right)$ is presented in Fig. 6.

With future precision of input data and reduction of theoretical uncertainty as in Case II, $\operatorname{Re} \epsilon, \Delta M_{B_{d}}$ and $\Delta M_{B_{s}}$ computed in the SM may deviate from the experimental values. Under this circumstance, it can be the case that theoretical calculations including the FCNH contributions are consistent with the experimental values. For example, suppose that the input parameters for $\operatorname{Re} \epsilon$ are precisely determined without changing their central values. A region of interest is then $m_{H} \sim 100 \mathrm{TeV}$ and $s_{c} s_{t}=-1$, because the central value of $\operatorname{Re} \epsilon$ is consistent with the experimental value in Fig. 5. If the model prediction for $\Delta M_{B_{d}}$ and $\Delta M_{B_{s}}$ should converge to the current central values, then, for $m_{H}=100 \mathrm{TeV}$ and $s_{c} s_{t}=-1$, the prediction and the experimental value of $\Delta M_{B_{d}}$ would be separated by more than $2 \sigma$ whereas the prediction for $\Delta M_{B_{s}}$ would be consistent with the experiment. However, it is possible that theoretical calculations of $\Delta M_{B_{d}}$ and $\Delta M_{B_{s}}$ converge to different values, independently of each other and of $\operatorname{Re} \epsilon$. If the theoretical inputs for $B_{d}^{0}-\bar{B}_{d}^{0}$ and $B_{s}^{0}-\bar{B}_{s}^{0}$ are determined as

$$
\begin{aligned}
& f_{B_{d}} \sqrt{B_{1}^{\mathrm{d} V L L}}\left(m_{b}\right)=158.5 \mathrm{MeV}, \\
& f_{B_{s}} \sqrt{B_{1}^{\mathrm{s} V L L}}\left(m_{b}\right)=211.9 \mathrm{MeV},
\end{aligned}
$$

$\Delta M_{B_{d}}$ and $\Delta M_{B_{s}}$ will be in agreement with the experiment. In this case, the correlation among the discrepancies between measurements and SM calculations of $\operatorname{Re} \epsilon$, $\Delta M_{B_{d}}$ and $\Delta M_{B_{s}}$ hints at the semialigned 2HDM. The model is further corroborated by tininess or nonobservation of deviations in the other $\Delta F=2$ processes; for instance, for Case I with $m_{H}=100 \mathrm{TeV}$, FCNH contributions to $B_{d}-\bar{B}_{d}$ mixing is highly suppressed as $\operatorname{Arg}\left(1+M_{12}^{d \mathrm{FCNH}} / M_{12}^{d \mathrm{SM}}\right)=\left(9.7_{-1.5}^{+1.7}\right) \times 10^{-5}$. Such a severe suppression on the observables other than $\operatorname{Re} \epsilon, \Delta M_{B_{d}}$ and $\Delta M_{B_{s}}$ is because the left-right parity restricts the flavorchanging part of the FCNH Yukawa couplings to be in the form Eq. (27), and hence is characteristic to the semialigned $2 \mathrm{HDM}$.

In the following, we comment on other flavor-violating observables. Since a charged scalar exchange alters $b \rightarrow$ $\mathrm{Cl}^{-} \nu$ decay rate at tree level, this model might be able to address the anomaly in $R_{D^{(*)}}$. However, this is not the case because the absolute value of the $b c H^{-}$coupling is small up to $\sqrt{2}\left|V_{c b} m_{b}\right| / v \sim O\left(10^{-3}\right)$, which does not exceed the SM $b c W^{-}$coupling, $\left|V_{c b}\right| \sim \mathcal{O}\left(10^{-2}\right)$.

We examine the correction to $b \rightarrow s s \bar{d}$ decay. In the $\mathrm{SM}$, this proceeds via the box diagram and is highly suppressed by the Glashow-Iliopoulos-Maiani mechanism as $\mathrm{Br}^{\mathrm{SM}}[b \rightarrow s s \bar{d}]=\mathcal{O}\left(10^{-12}\right)$ [28,29]. For the corresponding exclusive mode, experimental searches have been performed [30] and recently, the LHCb collaboration has reported $\mathrm{Br}\left[B^{-} \rightarrow K^{-} K^{-} \pi^{+}\right]<1.1 \times 10^{-8}(90 \% \mathrm{CL})$ [31]. In Ref. [32], the FCNH contribution to the decay width is calculated in the Type-III $2 \mathrm{HDM}$ and is found to be 


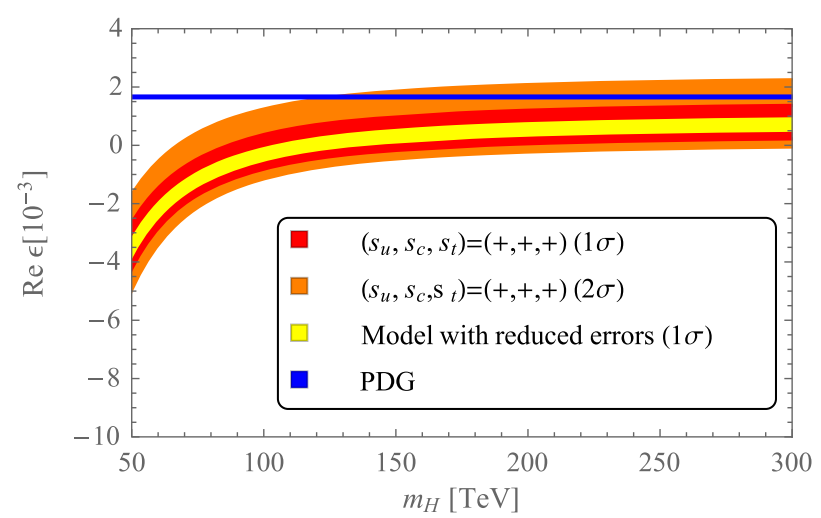

(a)

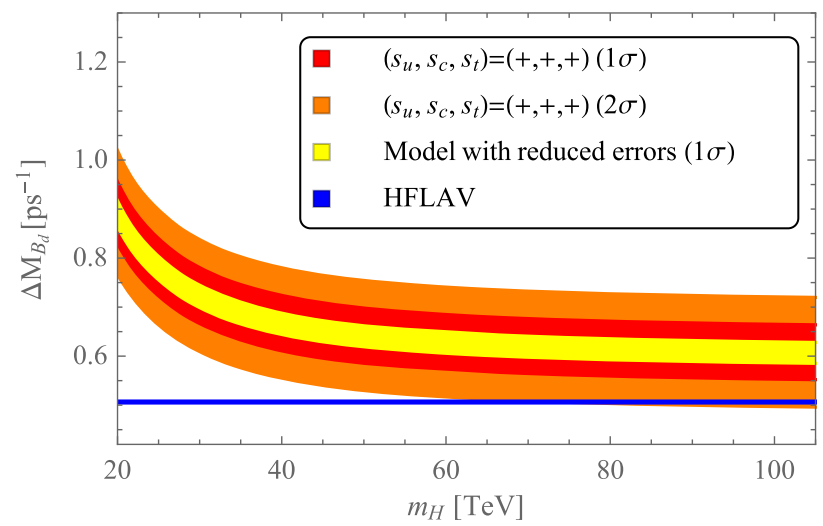

(c)

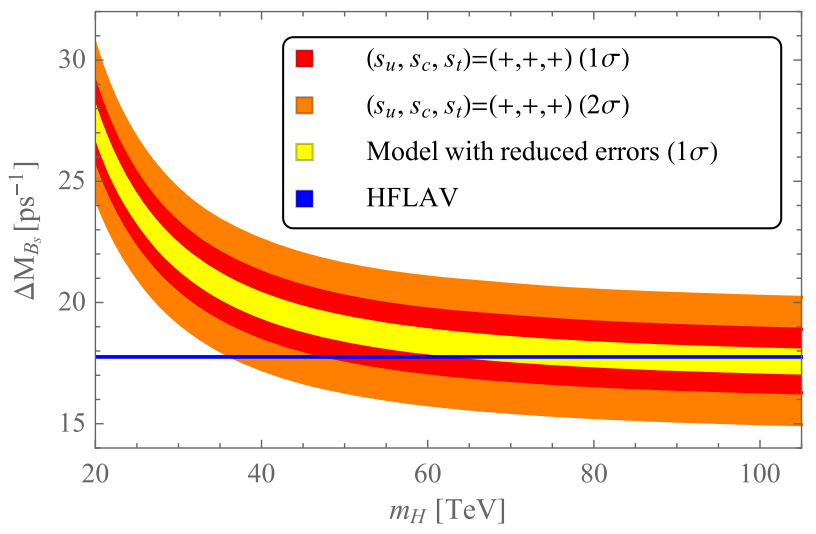

(e)

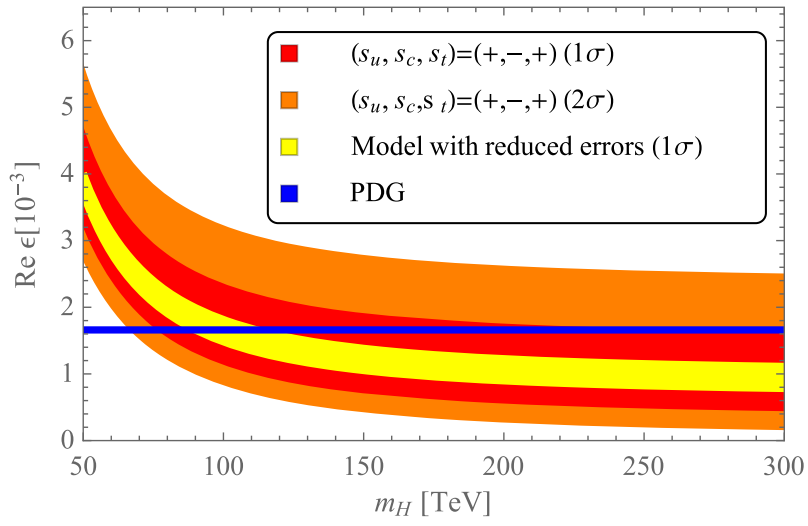

(b)

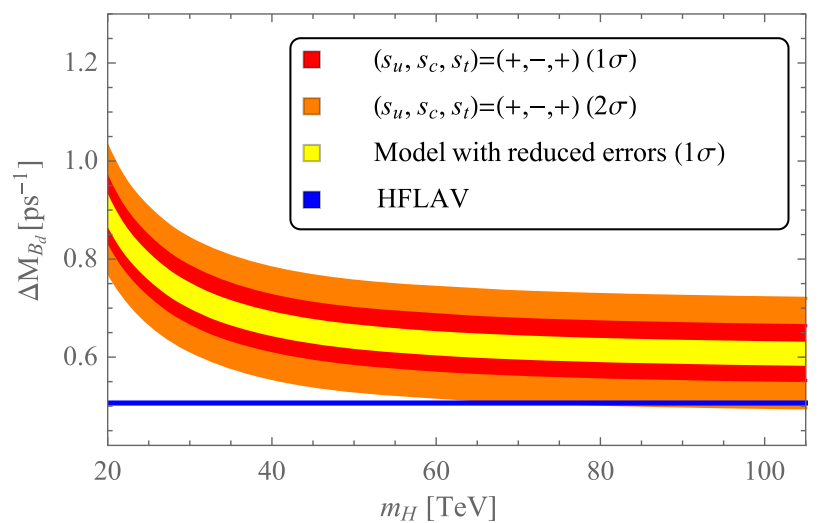

(d)

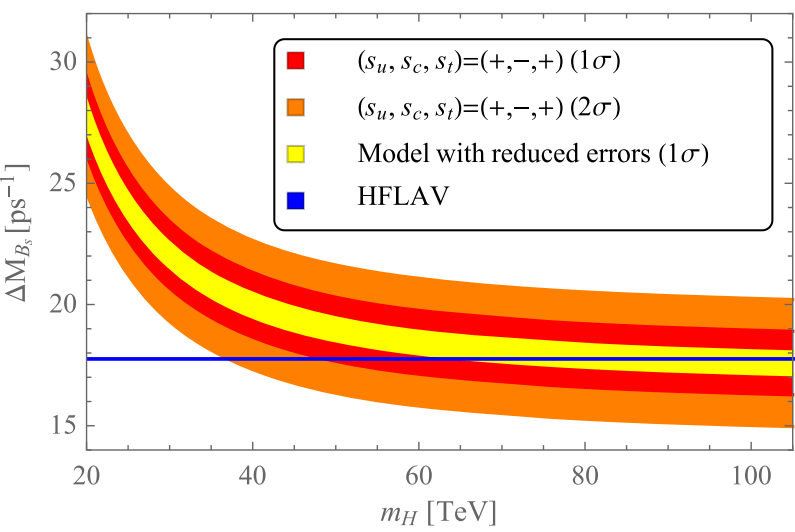

(f)

FIG. 6. Same figure as Fig. 5 in Case II.

$$
\begin{aligned}
\Gamma^{\mathrm{FCNH}}[b \rightarrow s s \bar{d}]= & \frac{m_{b}^{5}}{3072(2 \pi)^{3}}\left|\sum_{i, j}^{u, c, t} \lambda_{i}^{b d} \lambda_{j}^{s d} \frac{\left(m_{u}^{D}\right)^{i}\left(m_{u}^{D}\right)^{j}}{v^{2}}\right|^{2} \\
& \times\left[11\left(\frac{1}{m_{H}^{4}}+\frac{1}{m_{A}^{4}}\right)+\frac{2}{m_{H}^{2} m_{A}^{2}}\right]
\end{aligned}
$$

Using the above formula, we verify that the correction to $b \rightarrow s s \bar{d}$ decay in the semialigned $2 \mathrm{HDM}$ is given by
$\mathrm{Br}^{\mathrm{FCNH}}[b \rightarrow s s \bar{d}]=\mathcal{O}\left(10^{-14}-10^{-15}\right)$ for $m_{H}=10 \mathrm{TeV}$, which is much smaller than the SM prediction.

\section{SUMMARY AND DISCUSSION}

We have focused on a situation where the left-right symmetric model with the left-right parity is probed only through quark flavor changing neutral currents mediated by the heavy neutral scalar particles [flavor changing neutral 
Higgses (FCNHs)] arising from the $S U(2)_{L} \times S U(2)_{R}$ bifundamental scalar. We have extracted the bifundamental scalar part of the left-right model, named it "semialigned two Higgs doublet model," and investigated its phenomenology by focusing on various $\Delta F=2$ processes.

First, we have derived the current lower bound on the mass of FCNH, by calculating $\Delta M_{B_{d}}, \Delta M_{B_{s}}, \operatorname{Re} \epsilon$ and $\sin 2 \beta_{\text {eff }}$ with the inclusion of the FCNH exchange contribution, and fitting them and other observables with the CKM matrix. The tension in the fitting, represented by p-values, has given the bounds on the FCNH mass, which read: $m_{H}>75-84 \mathrm{TeV}$ for $3 \sigma$ and $m_{H}>56-64 \mathrm{TeV}$ for $5 \sigma$.

Second, we have made a prediction for $\left(\operatorname{Re} \epsilon, \Delta M_{B_{d}}\right.$, $\Delta M_{B_{s}}$ ) in terms of only one free parameter, i.e. the FCNH mass, under the assumption that uncertainties in the measurement of $\left|V_{u b}\right|$ and $\gamma$ are reduced from the current estimates, allowing us to determine the CKM matrix without being affected by the FCNH exchange. We have revealed that if the precision of $\left|V_{u b}\right|$ and $\gamma$ is improved and the SM prediction disagrees with the experimental data in such a way that the inclusion of FCNH contributions (for some unique value of the FCNH mass) is mandatory to fit the data, it hints at the semialigned two Higgs doublet model and the left-right model. In this case, the correlation among the discrepancies in measurements and SM calculations of $\left(\operatorname{Re} \epsilon, \Delta M_{B_{d}}, \Delta M_{B_{s}}\right)$ is considered as a sign of the model. Also, smallness or nonobservation of anomalies in the other $\Delta F=2$ observables supports the model, since it reflects the strong restriction on the FCNH Yukawa couplings by the left-right parity.

Finally, we comment that with a future $100 \mathrm{TeV}$ collider, it may be possible to directly produce $\mathrm{FCNHs}(H, A)$ and measure their decay branching ratios. The semialigned two Higgs doublet model predicts that the Yukawa couplings of $H, A$ to quarks are given by Eq. (25), and we further expect $\sin 2 \beta \ll 1$ because we need $\tan \beta \sim m_{t} / m_{b}$ to naturally realize the top and bottom Yukawa couplings. Therefore, if the branching ratios of $H, A$ obey the following rule,

$$
\begin{aligned}
& \operatorname{Br}(H \rightarrow \bar{t} t): \operatorname{Br}(H \rightarrow \bar{b} b)=\operatorname{Br}(A \rightarrow \bar{t} t): \\
& \operatorname{Br}(A \rightarrow \bar{b} b) \simeq m_{b}^{2}: m_{t}^{2}
\end{aligned}
$$

TABLE III. Full field content of the left-right symmetric model. $i=1,2,3$ is the flavor index.

\begin{tabular}{lcccc}
\hline \hline Field & Lorentz & & & \\
& $S O(1,3)$ & $S U(3)_{C}$ & $S U(2)_{L} \times S U(2)_{R}$ & $U(1)_{B-L}$ \\
\hline$q_{L}^{i}$ & $(\mathbf{2}, \mathbf{1})$ & $\mathbf{3}$ & $(\mathbf{2}, \mathbf{1})$ & $1 / 3$ \\
$q_{R}^{i}$ & $(\mathbf{1}, \mathbf{2})$ & $\mathbf{3}$ & $(\mathbf{1}, \mathbf{2})$ & $1 / 3$ \\
$\ell_{L}^{i}$ & $(\mathbf{2}, \mathbf{1})$ & $\mathbf{1}$ & $(\mathbf{2}, \mathbf{1})$ & -1 \\
$\ell_{R}^{i}$ & $(\mathbf{1}, \mathbf{2})$ & $\mathbf{1}$ & $(\mathbf{1}, \mathbf{2})$ & -1 \\
$\Phi$ & $\mathbf{1}$ & $\mathbf{1}$ & $(\mathbf{2}, \mathbf{2})$ & 0 \\
$\Delta_{L}$ & $\mathbf{1}$ & $\mathbf{1}$ & $\mathbf{( 3 , 1 )}$ & 2 \\
$\Delta_{R}$ & $\mathbf{1}$ & $\mathbf{1}$ & $\mathbf{( 1 , ~ 3 )}$ & 2 \\
\hline \hline
\end{tabular}

in accord with Eq. (25) with $\sin 2 \beta \ll 1$, then this is strong evidence for the semialigned two Higgs doublet model. A detailed collider study about the bifundamental scalar part of the left-right model is found in Ref. [8].

\section{ACKNOWLEDGMENTS}

We would like to thank Akimasa Ishikawa, who commented on future experimental precisions of CKM matrix elements and $\gamma$. This work is partially supported by Scientific Grants by the Ministry of Education, Culture, Sports, Science and Technology of Japan (Nos. 24540272, 26247038, 15H01037, 16H00871, and 16H02189).

\section{APPENDIX: LEFT-RIGHT SYMMETRIC MODEL WITH LEFT-RIGHT PARITY}

We present the left-right symmetric model [1] with the left-right parity. The gauge symmetry is $S U(3)_{C} \times S U(2)_{L} \times$ $S U(2)_{R} \times U(1)_{B-L}$, and the full field content is shown in Table III. The model is invariant under the left-right parity transformation, in which $S U(2)_{L}$ and $S U(2)_{R}$ gauge groups are interchanged and the fields transform as

$$
\Phi \leftrightarrow \Phi^{\dagger}, \quad \Delta_{L} \leftrightarrow \Delta_{R}, \quad q_{L} \leftrightarrow q_{R}, \quad \ell_{L} \leftrightarrow \ell_{R} .
$$

We write the bifundamental scalar $\Phi$ and triplet scalars $\Delta_{L}, \Delta_{R}$ as $2 \times 2$ matrices that transform under a $S U(2)_{L} \times S U(2)_{R}$ gauge transformation as

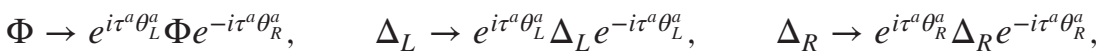

$$
\begin{aligned}
& \theta_{L}^{a}, \theta_{R}^{a} \text { : gauge parameters, } \quad \tau^{a} \equiv \sigma^{a} / 2 \text {. }
\end{aligned}
$$

The Yukawa couplings and the scalar potential of the left-right symmetric model with the left-right parity are found to be

$$
\begin{aligned}
-\mathcal{L}= & \left(Y_{q}\right)_{i j} \bar{q}_{L}^{i} \Phi q_{R}^{j}+\left(\tilde{Y}_{q}\right)_{i j} \bar{q}_{L}^{i} \tilde{\Phi} q_{R}^{j}+\left(Y_{\ell}\right)_{i j} \bar{\ell}_{L}^{i} \Phi \ell_{R}^{j}+\left(\tilde{Y}_{\ell}\right)_{i j} \bar{\ell}_{L}^{i} \tilde{\Phi} \ell_{R}^{j}+\text { H.c. } \\
& +\left(Y_{M}\right)_{i j}\left(\ell_{L}^{i T} \epsilon \Delta_{L} \ell_{L}^{j}+\ell_{R}^{i T} \epsilon \Delta_{R} \ell_{R}^{j}\right)+\text { H.c. }
\end{aligned}
$$




$$
\begin{aligned}
& +\mu_{1}^{2} \operatorname{tr}\left[\Phi^{\dagger} \Phi\right]+\mu_{2}^{2} \operatorname{tr}\left[\Phi^{\dagger} \tilde{\Phi}+\Phi \tilde{\Phi}^{\dagger}\right]+\mu_{3}^{2} \operatorname{tr}\left[\Delta_{L}^{\dagger} \Delta_{L}+\Delta_{R}^{\dagger} \Delta_{R}\right] \\
& +\lambda_{1} \operatorname{tr}\left[\Phi^{\dagger} \Phi\right]^{2}+\lambda_{2}\left(\operatorname{tr}\left[\Phi^{\dagger} \tilde{\Phi}\right]^{2}+\operatorname{tr}\left[\Phi \tilde{\Phi}^{\dagger}\right]^{2}\right)+\lambda_{3} \operatorname{tr}\left[\Phi^{\dagger} \tilde{\Phi}\right] \operatorname{tr}\left[\Phi \tilde{\Phi}^{\dagger}\right]+\lambda_{4} \operatorname{tr}\left[\Phi^{\dagger} \Phi\right] \operatorname{tr}\left[\Phi^{\dagger} \tilde{\Phi}+\Phi \tilde{\Phi}^{\dagger}\right] \\
& +\rho_{1}\left(\operatorname{tr}\left[\Delta_{L}^{\dagger} \Delta_{L}\right]^{2}+\operatorname{tr}\left[\Delta_{R}^{\dagger} \Delta_{R}\right]^{2}\right)+\rho_{2}\left(\operatorname{tr}\left[\Delta_{L} \Delta_{L}\right] \operatorname{tr}\left[\Delta_{L}^{\dagger} \Delta_{L}^{\dagger}\right]+\operatorname{tr}\left[\Delta_{R} \Delta_{R}\right] \operatorname{tr}\left[\Delta_{R}^{\dagger} \Delta_{R}^{\dagger}\right]\right) \\
& +\rho_{3} \operatorname{tr}\left[\Delta_{L}^{\dagger} \Delta_{L}\right] \operatorname{tr}\left[\Delta_{R}^{\dagger} \Delta_{R}\right]+\rho_{4}\left(\operatorname{tr}\left[\Delta_{L} \Delta_{L}\right] \operatorname{tr}\left[\Delta_{R}^{\dagger} \Delta_{R}^{\dagger}\right]+\operatorname{tr}\left[\Delta_{R} \Delta_{R}\right] \operatorname{tr}\left[\Delta_{L}^{\dagger} \Delta_{L}^{\dagger}\right]\right)+\alpha_{1} \operatorname{tr}\left[\Phi^{\dagger} \Phi\right] \operatorname{tr}\left[\Delta_{L}^{\dagger} \Delta_{L}+\Delta_{R}^{\dagger} \Delta_{R}\right] \\
& \left.+\alpha_{2 R} \operatorname{tr}\left[\Phi^{\dagger} \tilde{\Phi}\right] \operatorname{tr}\left[\Delta_{L}^{\dagger} \Delta_{L}\right]+\operatorname{tr}\left[\Phi \tilde{\Phi}^{\dagger}\right] \operatorname{tr}\left[\Delta_{R}^{\dagger} \Delta_{R}\right]+\operatorname{tr}\left[\Phi \tilde{\Phi}^{\dagger}\right] \operatorname{tr}\left[\Delta_{L}^{\dagger} \Delta_{L}\right]+\operatorname{tr}\left[\Phi^{\dagger} \tilde{\Phi}\right] \operatorname{tr}\left[\Delta_{R}^{\dagger} \Delta_{R}\right]\right) \\
& +i \alpha_{2 I}\left(\operatorname{tr}\left[\Phi^{\dagger} \tilde{\Phi}\right] \operatorname{tr}\left[\Delta_{L}^{\dagger} \Delta_{L}\right]+\operatorname{tr}\left[\Phi \tilde{\Phi}^{\dagger}\right] \operatorname{tr}\left[\Delta_{R}^{\dagger} \Delta_{R}\right]-\operatorname{tr}\left[\Phi \tilde{\Phi}^{\dagger}\right] \operatorname{tr}\left[\Delta_{L}^{\dagger} \Delta_{L}\right]-\operatorname{tr}\left[\Phi^{\dagger} \tilde{\Phi}\right] \operatorname{tr}\left[\Delta_{R}^{\dagger} \Delta_{R}\right]\right)+\alpha_{3} \operatorname{tr}\left[\Phi \Phi^{\dagger} \Delta_{L}^{\dagger} \Delta_{L}+\Phi^{\dagger} \Phi \Delta_{R}^{\dagger} \Delta_{R}\right] \\
& +\beta_{1}\left[\Phi \Delta_{R} \Phi^{\dagger} \Delta_{L}^{\dagger}+\Phi^{\dagger} \Delta_{L} \Phi \Delta_{R}^{\dagger}\right]+\beta_{2}\left[\tilde{\Phi} \Delta_{R} \Phi^{\dagger} \Delta_{L}^{\dagger}+\tilde{\Phi}^{\dagger} \Delta_{L} \Phi \Delta_{R}^{\dagger}\right]+\beta_{3}\left[\Phi \Delta_{R} \tilde{\Phi}^{\dagger} \Delta_{L}^{\dagger}+\Phi^{\dagger} \Delta_{L} \tilde{\Phi} \Delta_{R}^{\dagger}\right] \\
& \text { with } \tilde{\Phi} \equiv i \sigma_{2} \Phi^{*} i \sigma_{2},
\end{aligned}
$$

where $Y_{q}, \tilde{Y}_{q}, Y_{\ell}, \tilde{Y}_{\ell}$ are Hermitian matrices, and $Y_{M}$ is a complex-valued symmetric matrix. The mass terms $\mu_{1}^{2}, \mu_{2}^{2}$, $\mu_{3}^{2}$ and the coupling constants $\lambda_{1}, \lambda_{2}, \lambda_{3}, \lambda_{4}, \rho_{1}, \rho_{2}, \rho_{3}, \rho_{4}$, $\alpha_{1}, \alpha_{2}, \tilde{\alpha}_{2}, \alpha_{3}, \beta_{1}, \beta_{2}, \beta_{3}$ are all real.

Through a $S U(2)_{R} \times U(1)_{B-L}$ symmetry transformation, one can set the VEV of $\Delta_{R}$ in the following form:

$$
\left\langle\Delta_{R}\right\rangle=\frac{1}{\sqrt{2}}\left(\begin{array}{cc}
0 & 0 \\
v_{R} & 0
\end{array}\right), \quad v_{R}>0 .
$$

Through a subsequent $S U(2)_{L}$ and $\sigma_{3}$ part of $S U(2)_{R}$ symmetry transformation, one can set

$$
\langle\Phi\rangle=\left(\begin{array}{cc}
v_{1} & 0 \\
0 & -e^{i \alpha} v_{2}
\end{array}\right), \quad v_{1}>0, \quad v_{2}>0,
$$

which gives rise to a phase for the $\Delta_{R} \mathrm{VEV}$, but this can be negated by a $U(1)_{B-L}$ symmetry transformation.
[1] J. C. Pati and A. Salam, Lepton number as the fourth color, Phys. Rev. D 10, 275 (1974); 11, 703(E) (1975); R. N. Mohapatra and J. C. Pati, A natural left-right symmetry, Phys. Rev. D 11, 2558 (1975); G. Senjanovic and R. N. Mohapatra, Exact left-right symmetry and spontaneous violation of parity, Phys. Rev. D 12, 1502 (1975).

[2] A. Maiezza, M. Nemevsek, F. Nesti, and G. Senjanovic, Left-right symmetry at LHC, Phys. Rev. D 82, 055022 (2010).

[3] S. Bertolini, A. Maiezza, and F. Nesti, Present and future K and $\mathrm{B}$ meson mixing constraints on $\mathrm{TeV}$ scale left-right symmetry, Phys. Rev. D 89, 095028 (2014).

[4] R. N. Mohapatra, G. Senjanovic, and M. D. Tran, Strangeness changing processes and the limit on the right-handed gauge boson mass, Phys. Rev. D 28, 546 (1983); F. J. Gilman and M. H. Reno, Restrictions on left-right symmetric gauge theories from the neutral kaon system and $B$ decays, Phys. Lett. 127B, 426 (1983); Restrictions from the neutral $K$ and $B$ meson systems on left-right symmetric gauge theories, Phys. Rev. D 29, 937 (1984); G. Ecker, W. Grimus, and H. Neufeld, Higgs induced flavor changing neutral interactions in $S U(2)_{L} \times S U(2)_{R} \times U(1)$, Phys. Lett. 127B, 365 (1983); 132B, 467(E) (1983); G. Ecker and W. Grimus, $C P$ violation and left-right symmetry, Nucl. Phys. B258, 328 (1985); Mass mixing, $C P$ violation and left-right symmetry for heavy neutral mesons, Z. Phys. C 30, 293 (1986); M. E. Pospelov, FCNC in left-right symmetric theories and constraints on the right-handed scale, Phys. Rev. D 56, 259 (1997).

[5] Y. Zhang, H. An, X. Ji, and R. N. Mohapatra, General CP violation in minimal left-right symmetric model and constraints on the right-handed scale, Nucl. Phys. B802, 247 (2008).

[6] J. Chakrabortty, J. Gluza, T. Jelinski, and T. Srivastava, Theoretical constraints on masses of heavy particles in leftright symmetric models, Phys. Lett. B 759, 361 (2016).

[7] A. Maiezza, M. Nemevsek, and F. Nesti, Perturbativity and mass scales in the minimal left-right symmetric model, Phys. Rev. D 94, 035008 (2016); A. Maiezza, G. Senjanovic, and J. C. Vasquez, Higgs sector of the minimal left-right symmetric theory, Phys. Rev. D 95, 095004 (2017).

[8] P. S. B. Dev, R. N. Mohapatra, and Y. Zhang, Probing the Higgs sector of the minimal left-right symmetric model at future hadron colliders, J. High Energy Phys. 05 (2016) 174.

[9] A. Maiezza and M. Nemevsek, Strong P invariance, neutron electric dipole moment, and minimal left-right parity at LHC, Phys. Rev. D 90, 095002 (2014).

[10] R. Kuchimanchi, P/CP conserving CP/P violation solves strong CP problem, Phys. Rev. D 82, 116008 (2010); Maximal $C P$ and bounds on the neutron electric dipole moment from $\mathrm{P}$ and $C P$ breaking, Phys. Rev. D 86, 036002 (2012); Leptonic $C P$ problem in left-right symmetric model, Phys. Rev. D 91, 071901 (2015). 
[11] C. A. Baker et al., An Improved Experimental Limit on the Electric Dipole Moment of the Neutron, Phys. Rev. Lett. 97, 131801 (2006); J. M. Pendlebury et al., Revised experimental upper limit on the electric dipole moment of the neutron, Phys. Rev. D 92, 092003 (2015).

[12] J. Charles et al., Current status of the standard model CKM fit and constraints on $\Delta F=2$ new physics, Phys. Rev. D 91, 073007 (2015).

[13] A. J. Buras, S. Jager, and J. Urban, Master formulae for $\Delta F=2$ NLO QCD factors in the standard model and beyond, Nucl. Phys. B605, 600 (2001).

[14] T. Inami and C. S. Lim, Effects of superheavy quarks and leptons in low-energy weak processes $K_{L} \rightarrow \mu \bar{\mu}, K^{+} \rightarrow$ $\pi^{+} \nu \bar{\nu}$ and $K^{0} \leftrightarrow \bar{K}^{0}$, Prog. Theor. Phys. 65, 297 (1981); 65, 1772(E) (1981).

[15] S. Herrlich and U. Nierste, Enhancement of the $K_{L}-K_{S}$ mass difference by short distance QCD corrections beyond leading logarithms, Nucl. Phys. B419, 292 (1994); Indirect $C P$ violation in the neutral kaon system beyond leading logarithms, Phys. Rev. D 52, 6505 (1995); The Complete $|\Delta S|=2$ Hamiltonian in the next-to-leading order, Nucl. Phys. B476, 27 (1996).

[16] A. J. Buras, M. Misiak, and J. Urban, Two loop QCD anomalous dimensions of flavor changing four quark operators within and beyond the standard model, Nucl. Phys. B586, 397 (2000).

[17] C. Patrignani et al. (Particle Data Group), Review of particle physics, Chin. Phys. C 40, 100001 (2016).

[18] N. Carrasco, P. Dimopoulos, R. Frezzotti, V. Lubicz, G. C. Rossi, S. Simula, and C. Tarantino (ETM Collaboration), $\Delta S=2$ and $\Delta C=2$ bag parameters in the standard model and beyond from $\mathrm{N}_{f}=2+1+1$ twisted-mass lattice QCD, Phys. Rev. D 92, 034516 (2015).

[19] N. Carrasco et al. (ETM Collaboration), B-physics from $N_{f}=2$ tmQCD: The standard model and beyond, J. High Energy Phys. 03 (2014) 016.

[20] Y. Amhis et al., Averages of $b$-hadron, $c$-hadron, and $\tau$-lepton properties as of summer 2016, Eur. Phys. J. C 77, 895 (2017).
[21] See review, e.g., T. Gershon and Y. Nir, $C P$ violation in the quark sector in Ref. [17].

[22] H.n. Li and S. Mishima, Penguin pollution in the $B^{0} \rightarrow J / \psi K_{S}$ decay, J. High Energy Phys. 03 (2007) 009.

[23] P. Ball, J. M. Frere, and J. Matias, Anatomy of mixing induced $C P$ asymmetries in left-right symmetric models with spontaneous $C P$ violation, Nucl. Phys. B572, 3 (2000).

[24] K. Kiers, J. Kolb, J. Lee, A. Soni, and G. H. Wu, Ubiquitous $C P$ violation in a top inspired left-right model, Phys. Rev. D 66, 095002 (2002).

[25] L. Wolfenstein, Parametrization of the Kobayashi-Maskawa Matrix, Phys. Rev. Lett. 51, 1945 (1983).

[26] A. J. Buras, M. E. Lautenbacher, and G. Ostermaier, Waiting for the top quark mass, $K^{+} \rightarrow \pi^{+} \nu \bar{\nu}, B_{s}^{0}-\bar{B}_{s}^{0}$ mixing and $C P$ asymmetries in $B$ decays, Phys. Rev. D 50, 3433 (1994).

[27] A. Hocker, H. Lacker, S. Laplace, and F. Le Diberder, A new approach to a global fit of the CKM matrix, Eur. Phys. J. C 21, 225 (2001).

[28] K. Huitu, D. X. Zhang, C. D. Lu, and P. Singer, Searching for New Physics in $b \rightarrow s s \bar{d}$ Decays, Phys. Rev. Lett. 81, 4313 (1998).

[29] C. D. Lü, F. Munir, and Q. Qin, $b \rightarrow s s \bar{d}$ decay in RandallSundrum models, Chin. Phys. C 41, 053106 (2017).

[30] G. Abbiendi et al. (OPAL Collaboration), Search for new physics in rare B decays, Phys. Lett. B 476, 233 (2000); A. Garmash et al. (Belle Collaboration), Study of $B$ meson decays to three body charmless hadronic final states, Phys. Rev. D 69, 012001 (2004); B. Aubert et al. (BABAR Collaboration), Search for the highly suppressed decays $B^{-} \rightarrow K^{+} \pi^{-} \pi^{-}$and $B^{-} \rightarrow K^{-} K^{-} \pi^{+}$, Phys. Rev. D 78, 091102 (2008).

[31] R. Aaij et al. (LHCb Collaboration), Search for the suppressed decays $B^{+} \rightarrow K^{+} K^{+} \pi^{-}$and $B^{+} \rightarrow \pi^{+} \pi^{+} K^{-}$, Phys. Lett. B 765, 307 (2017).

[32] K. Huitu and C. D. Lü, P. Singer, and D. X. Zhang, $b \rightarrow s s \bar{d}$ decay in two Higgs doublet models, Phys. Lett. B 445, 394 (1999). 